\title{
Reduced basis method for linear elasticity problems with many parameters*
}

\author{
Roberto Milani ${ }^{\sharp}, \dagger$ Alfio Quarteroni ${ }^{\sharp, \S}$, Gianluigi Rozza ${ }^{\S, \ddagger}$ \\ May 20, 2008 \\ \# MOX- Modellistica e Calcolo Scientifico \\ Dipartimento di Matematica "F. Brioschi" \\ Politecnico di Milano \\ via Bonardi 9, 20133 Milano, Italy \\ alfio.quarteroni@mate.polimi.it \\ $\S$ Modeling and Scientific Computing, IACS-CMCS \\ École Polytechnique Fédérale de Lausanne, EPFL \\ Station 8, CH-1015 Lausanne, Switzerland \\ \{alfio.quarteroni, gianluigi.rozza\}@epfl.ch
}

Keywords: reduced basis method, Galerkin approximation, finite element method, parametrized partial differential equations, linear elasticity, plane stress and plane strain approximation, isotropic and orthotropic material, optimization.

\begin{abstract}
The reduced basis $(\mathrm{RB})$ methods are proposed here for the solution of parametrized equations in linear elasticity problems. The fundamental idea underlying RB methods is to decouple the generation and projection stages (offline/online computational procedures) of the approximation process in order to solve parametrized equations in a rapid, inexpensive and reliable way.

The method allows important computational savings with respect to the classical Galerkinfinite element method, ill suited to a repetitive environment like the parametrized contexts of optimization, many queries and sensitivity analysis. We consider different parametrization for the systems: either physical quantities -to model the materials and loadsand geometrical parameters -to model different geometrical configurations--. Then we
\end{abstract}

*This work has been supported by Italian MIUR Cofin2005 "Numerical modeling for scientific computing and advanced applications", EPFL and Swiss National Science Foundation.

${ }^{\dagger}$ Currently structural engineer at Tecnomare SPA, ENI Group, roberto.milani@tecnomare.it

${ }^{\ddagger}$ Currently at MIT, Massachusetts Institute of Technology, Mechanical Engineering Department, 77 Mass Avenue, Room 3-264, 02139 Cambridge MA, USA, rozza@mit.edu 
describe three different applications of the method in problems with isotropic and orthotropic materials working in plane stress and plane strain approximation and subject to harmonic loads.

\section{Introduction and motivation}

In many systems governed by partial differential equations (PDE), the most important quantities of interest are not the full field variable, but rather outputs that describe the characteristic features of the system, such as energies, forces, temperatures, fluxes or critical stresses/strains. These outputs are controlled by parameters, or inputs, that describe the configuration of the system, or else by components, such as geometry, properties, loads or environment settings. The relevant system behavior is thus described by an implicit inputoutput relationship, or a mathematical description of the physical problem. As the physical problem becomes more complex, the computational time may increase prohibitively; in some cases, it is even impossible to provide enough resources to store the necessary data. The current computational methods may thus prove inadequate, especially in those contexts requiring real-time response or many queries, for example, engineering optimization and adaptive design, parameter estimation or feedback control of processes.

The reduced basis $(\mathrm{RB})$ method is a computational procedure that provides both fast and reliable evaluation of an input-output relationship. The main ingredients are $(i)$ rapid uniformly convergent reduced basis approximations [16]; $(i i)$ the possibility to use a posteriori error estimation [25] which provides sharp and rigorous bounds for the error in the output and (iii) offline/online computational strategy which allows rapid calculation of both output approximation and associated error bound. The result is straightforward: an output calculation method which has both the accuracy of a "full" numerical simulation model (say, using the finite element method) and inexpensive computational cost. Besides, the development of a posteriori error estimators is another key ingredient which leads to minimize the computational cost while ensuring the achievement of a sufficient accuracy.

The reduced basis approximation has been first introduced in the late 1970s [17, 20, 21, $22,23]$ for complex nonlinear structural analysis, but only recently it has received a lot of attention and has been analyzed from a methodological point of view, and developed to be applied to a much larger class of parametrized PDEs (with emphasis on reliability, efficiency, accuracy and convergence), - in particular, linear and nonlinear elliptic PDEs with affine and nonaffine parametric dependence - relevant to many engineering applications. In the elasticity field see, for example, recent works such as $[18,33,12,10]$. For a wider perspective on RB see [24].

The goal of this paper is the extension of RB method to problems arising in linear elasticity with many parameters of different nature (either physical and geometrical) representing different materials, configurations and loads. We apply RB methods to approximate problems using plane stress and plane strain formulation [31] and to deal both with isotropic and orthotropic materials, for example composites [3], by parametrizing also the constitutive law for the material. 
The paper is organized as follows. In the next Section we introduce an abstract formulation for a general parametrized problem and the approximation of its solution and an output of interest. Then in Section 3 we briefly recall the reduced basis methodology, based on our "truth" finite element approximation, and its ingredients and properties. In Section 4 we recall the mathematical formulation of linear elasticity problems, in Sections 5, 6 and 7 we present several model problems as examples: numerical results are discussed and compared to theoretical and other numerical results already available whenever possible. In particular in Section 5 we present a bi-material rod working in plane strain formulation and made up of isotropic material; then in Section 6 we deal with an orthotropic lamina and then composite materials in plane stress formulation. In Section 7 we discuss results dealing with a damaged isotropic lamina working in plane stress and under harmonic shear loads. Finally we discuss some future works and perspectives.

\section{Parametrized problems}

\subsection{Exact formulation}

We consider a polygonal domain $\Omega \subset \mathbb{R}^{d}$, with $d=1,2$ or 3 and a functional space $X$ with its associated scalar product $(., .)_{X}$ and norm $\|\cdot\|_{X}=(., .)^{1 / 2}$. We define a set of $P$ parameters $\mathcal{D}^{\mu} \subset \mathbb{R}^{P}$, whose elements (the parameters) are indicated with $\mu$. We introduce a parametrized bilinear, symmetric, continuous and coercive form $a: X \times X \times \mathcal{D}^{\mu} \rightarrow \mathbb{R}$ and the continuos linear forms $f: X \times \mathcal{D}^{\mu} \rightarrow \mathbb{R}$ and $l: X \times \mathcal{D}^{\mu} \rightarrow \mathbb{R}$.

A crucial property to deal in an efficient way with parametrized problems is the affine decomposition of the operator that governs our problem, so that we can rewrite the bilinear form $a(w, v ; \mu)$ as

$$
a(w, v ; \mu)=\sum_{q=1}^{Q} \sigma^{q}(\mu) a^{q}(w, v), \forall w, v \in X, \forall \mu \in \mathcal{D}^{\mu}
$$

for $q=1, \ldots, Q$ (not too large) parameter-dependent functions $\sigma^{q}: \mathcal{D}^{\mu} \rightarrow \mathbb{R}$, and parameterindependent forms $a^{q}: X \times X \rightarrow \mathbb{R}$.

The affine parameter decomposition is also applied to $f(v ; \mu)$ and $l(v ; \mu)$, but for simplicity we may consider $f(v)$ and $l(v)$. The abstract formulation of the problem is: $\forall \mu \in \mathcal{D}^{\mu}$, compute the output $s(\mu) \in \mathbb{R}$ with:

$$
s(\mu)=l(u(\mu))
$$

where $u(\mu) \in X$ is called the state variable and is in fact the solution of the variational problem

$$
a(u(\mu), v ; \mu)=f(v), \quad \forall v \in X .
$$

\subsection{Galerkin Approximation}

To compute the solution $u(\mu)$ of $(2)$ we first introduce a finite element discretization. For that, let $X_{h} \subset X$ be a finite element space of dimension $\mathcal{N}$ associated with a fine triangulation 
of our domain $\Omega$ [26]. The Galerkin-finite element approximation reads: $\forall \mu \in \mathcal{D}^{\mu}$, compute the output:

$$
s_{h}(\mu)=l\left(u_{h}(\mu)\right)
$$

where $u_{h} \in X_{h}$ is the solution of

$$
a\left(u_{h}(\mu), v ; \mu\right)=f(v), \quad \forall v \in X_{h} .
$$

We introduce a suitable basis for $X_{h}\left\{\varphi_{m}, m=1, \ldots, \mathcal{N}\right\}$, the associated symmetric and positive definite matrix $\underline{A}_{h}(\mu) \in \mathbb{R}^{\mathcal{N} \times \mathcal{N}}$, defined as:

$$
A_{h_{m, k}}=a\left(\varphi_{m}, \varphi_{k} ; \mu\right), \quad \forall m, k \in\{1, \ldots, \mathcal{N}\},
$$

and the discrete functionals $\underline{F}_{h}\left(\right.$ or $\left.\underline{L}_{h}\right) \in \mathbb{R}^{\mathcal{N}}$ as

$$
F_{h_{m}}=f\left(\varphi_{m}\right)\left(\text { or } L_{h_{m}}=l\left(\varphi_{m}\right)\right), \quad \forall m \in\{1, \ldots, \mathcal{N}\} .
$$

We can formulate the Galerkin problem as: for all $\mu \in \mathcal{D}^{\mu}$, compute the output:

$$
s_{h}(\mu)=\underline{L}_{h}^{T} \underline{u}_{h}(\mu)
$$

where $\underline{u}_{h}(\mu) \in \mathbb{R}^{\mathcal{N}}$ is the unique solution of

$$
\underline{A}_{h}(\mu) \underline{u}_{h}(\mu)=\underline{F}_{h} .
$$

Note that $u_{h}(\mu)$ and $\underline{u}_{h}(\mu)=\left(u_{h_{1}}, \ldots, u_{h_{\mathcal{N}}}\right)$ are related by the expression:

$$
u_{h}(\mu)=\sum_{m=1}^{\mathcal{N}} u_{h_{m}}(\mu) \varphi_{m}
$$

For simplicity we consider just "compliant" output, i.e. $l(u)=f(u)$.

\section{Reduced basis (RB) approximation}

We introduce a set of "snapshots" $S_{N}=\left\{\mu^{1}, \ldots, \mu^{N}\right\}$ where $\mu^{n} \in \mathcal{D}^{\mu}, n=1, \ldots, N$. For each $\mu^{n}$ we compute a finite element solution $u_{h}\left(\mu^{n}\right)$ in the space $X_{h}$ on a sufficiently fine mesh. We define the global reduced basis space as:

$$
W_{N}=\operatorname{span}\left\{\zeta^{n} \equiv u_{h}\left(\mu^{n}\right), n=1, \ldots, N\right\}
$$

In the RB formulation we look for an approximation $u_{N}(\mu)$ of $u_{h}(\mu)$ in $W_{N}$, given as:

$$
u_{N}(\mu)=\sum_{j=1}^{N} u_{N j}(\mu) \zeta^{j}=\left(\underline{u}_{N}(\mu)\right)^{T} \underline{\zeta},
$$


where $\underline{u}_{N}(\mu) \in \mathbb{R}^{N}$ is the vector of the "weights" $u_{N j}, j=1, \ldots, N ; u_{N}(\mu) \in W_{N}$ is the reduced basis solution, according to (9). The reduced basis approximation is: $\forall \mu \in \mathcal{D}^{\mu}$, compute $s_{N}(\mu)=l\left(u_{N}(\mu)\right)$, where $u_{N}(\mu) \in W_{N}$ is given by:

$$
a\left(u_{N}(\mu), v ; \mu\right)=f(v), \quad \forall v \in W_{N} .
$$

In Figure 1 we heuristically represent the main idea of the method: we suppose that every point on the line represents a different solution for a different $\mu^{n}$, the line represents the (smooth) manifold induced by the parametric dependence of the specific problem, this manifold is the subspace $W_{N} \subset X_{h}$ : a new and generic solution $u_{h}\left(\mu^{\text {new }}\right)$, for a new value $\mu^{\text {new }}$ of the parameter is gotten as a linear combination $\left(u_{h}\left(\mu^{1}\right), u_{h}\left(\mu^{2}\right), u_{h}\left(\mu^{3}\right), \ldots u_{h}\left(\mu^{N}\right)\right)$ of previously computed solutions.
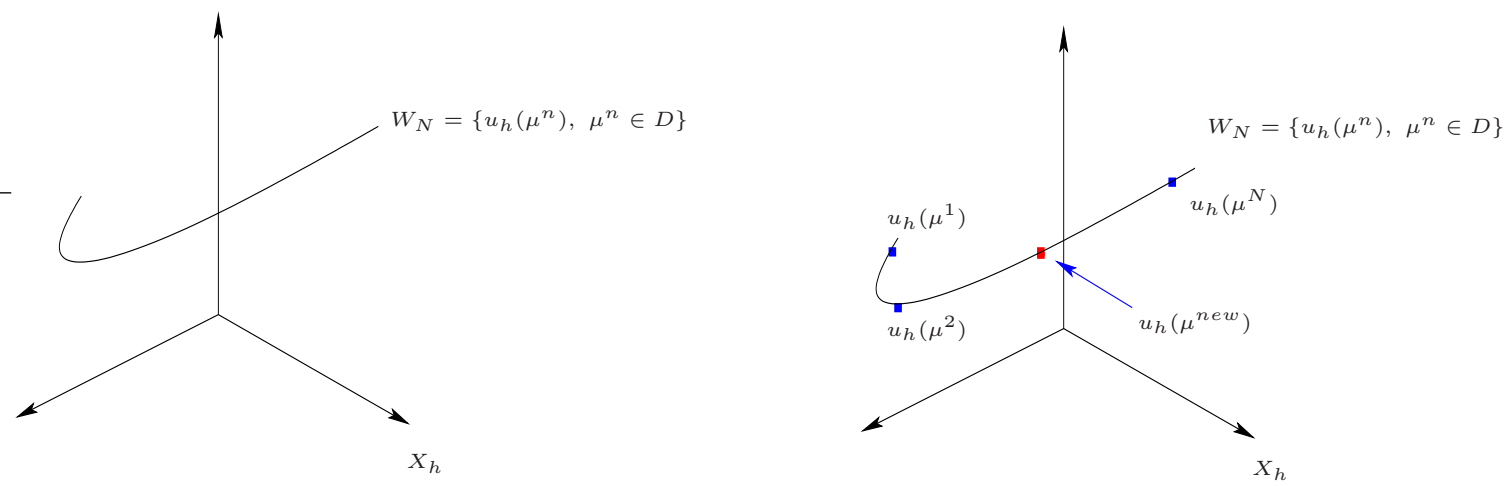

Figure 1: Heuristic idea of low dimensional manifold on which the parametrized solutions reside (left): the space $W_{N}$ made up of global selected approximation functions; solutions $u_{h}\left(\mu^{n}\right)$ for selected values of parameter $\mu$ on the manifold and $u_{h}\left(\mu^{n e w}\right)$ as combination of previously computed solutions (right).

By selecting as test functions the basis elements of $W_{N}$ components, i.e. $v=\zeta^{i}, i=$ $1, \ldots, N$ and replacing (9) in (10) we build the algebraic system to get $\underline{u}_{N}(\mu) \in \mathbb{R}^{N}$ :

$$
\underline{A}_{N}(\mu) \underline{u}_{N}(\mu)=\underline{F}_{N},
$$

and to compute the output

$$
s_{N}(\mu)=\underline{F}_{N}^{T} \underline{u}_{N}(\mu) .
$$

The elements of $\underline{A}_{N}(\mu) \in \mathbb{R}^{N \times N}$ are given by

$$
A_{N i, j}(\mu)=a\left(\zeta^{j}, \zeta^{i} ; \mu\right), \quad \forall i, j \in\{1, \ldots, N\} .
$$

As $\zeta^{i}$ is the finite element solution for a certain $\mu^{i}$, we can develop $\zeta^{i}$ in terms of $\varphi_{m}$, $m=1, \ldots, \mathcal{N}$ :

$$
\zeta^{i}=\sum_{m=1}^{\mathcal{N}} \zeta_{m}^{i} \varphi_{m}
$$


By replacing (14) in (13) we have:

$$
A_{N i, j}(\mu)=a\left(\sum_{m=1}^{\mathcal{N}} \zeta_{m}^{i} \varphi_{m}, \sum_{k=1}^{\mathcal{N}} \zeta_{k}^{j} \varphi_{k} ; \mu\right), \quad \forall i, j \in\{1, \ldots, N\}
$$

and thanks to the bilinearity of $a(., . ; \mu), \forall \mu \in \mathcal{D}^{\mu}$, we get:

$$
A_{N i, j}(\mu)=\sum_{m=1}^{\mathcal{N}} \sum_{k=1}^{\mathcal{N}} \zeta_{m}^{i} \zeta_{k}^{j} a\left(\varphi_{m}, \varphi_{k} ; \mu\right), \quad \forall i, j \in\{1, \ldots, N\} .
$$

Using a more compact formulation:

$$
A_{N i, j}(\mu)=\left(\underline{\zeta}^{i}\right)^{T} \underline{A}_{h}(\mu)\left(\underline{\zeta}^{j}\right), \quad \forall i, j \in\{1, \ldots, N\} .
$$

Indicating with $\underline{Z}=\left\{\zeta^{1}, \ldots, \zeta^{N}\right\}$ we write:

$$
\underline{A}_{N}(\mu)=\underline{Z}^{T} \underline{A}_{h}(\mu) \underline{Z} .
$$

Thanks to the affine decomposition of the operator, the bilinear form $a(., ., \mu)$ can be split as follows:

$$
a(., . ; \mu)=\sum_{q=1}^{Q} \sigma^{q}(\mu) a^{q}(., .)
$$

Consequently, we can get a great computational saving by decomposing

$$
\sum_{j=1}^{N} a\left(\zeta^{j}, \zeta^{i} ; \mu\right) u_{N j}=f\left(\zeta^{i}\right), \quad \forall i=1, \ldots, N
$$

into this form:

$$
\sum_{q=1}^{Q} \sum_{j=1}^{N} \sigma^{q}(\mu) a^{q}\left(\zeta^{j}, \zeta^{i}\right) u_{N j}=f\left(\zeta^{i}\right), \quad \forall i=1, \ldots, N .
$$

Then from (19) we get

$$
\begin{gathered}
A_{N i, j}(\mu)=\sum_{q=1}^{Q} \sigma^{q}(\mu) a^{q}\left(\zeta^{i}, \zeta^{j}\right)= \\
\sum_{q=1}^{Q} \sigma^{q}(\mu) a^{q}\left(\sum_{m=1}^{\mathcal{N}} \zeta_{m}^{i} \varphi_{m}, \sum_{k=1}^{\mathcal{N}} \zeta_{k}^{j} \varphi_{k}\right)=\sum_{q=1}^{Q} \sigma^{q}(\mu) \sum_{m=1}^{\mathcal{N}} \sum_{k=1}^{\mathcal{N}} \zeta_{m}^{i} \zeta_{k}^{j} a^{q}\left(\varphi_{m}, \varphi_{k}\right) \\
=\sum_{q=1}^{Q} \sigma^{q}(\mu)\left(\underline{\zeta}^{i}\right)^{T} \underline{A}_{h}^{q}\left(\underline{\zeta}^{j}\right)=\sum_{q=1}^{Q} \sigma^{q}(\mu) \underline{A}_{N i, j}^{q}, \quad \forall i, j \in\{1, \ldots, N\},
\end{gathered}
$$


The $Q$ matrices $\underline{A}_{N}^{q} \in \mathbb{R}^{N \times N}$ need to be computed only once since they are parameter independent,

$$
\underline{A}_{N}^{q}=\underline{Z}^{T} \underline{A}_{h}^{q} \underline{Z}, \quad q=1, \ldots, Q .
$$

Then the parametrized operator can be obtained by a simple assembling procedure:

$$
\underline{A}_{N}(\mu)=\sum_{q=1}^{Q} \sigma^{q}(\mu) \underline{A}_{N}^{q} .
$$

We adopt the same procedure for the right-hand-side:

$$
\begin{aligned}
F_{N i} & =f\left(\zeta^{i}\right)=f\left(\sum_{m=1}^{\mathcal{N}} \zeta_{m}^{i} \varphi_{m}\right) \\
& =\sum_{m=1}^{\mathcal{N}} \zeta_{m}^{i} f\left(\varphi_{m}\right)=\left(\underline{\zeta}^{i}\right)^{T} \underline{F}_{h}, \quad i=\{1, \ldots, N\}
\end{aligned}
$$

so that:

$$
\underline{F}_{N}=\underline{Z}^{T} \underline{F}_{h}
$$

The resulting $\mathrm{RB}$ problem

$$
\underline{A}_{N}(\mu) \underline{u}_{N}(\mu)=\underline{F}_{N},
$$

with $\underline{A}_{N}(\mu) \in \mathbb{R}^{N \times N}$ and $\underline{F}_{N} \in \mathbb{R}^{N}$, features matrices $\underline{A}_{N}(\mu)$ that are full, whereas those $\underline{A}_{h}(\mu)$ of the underlying finite element method are sparse. To solve (21) we will use direct methods [27], since the size of matrix $N$ is in general small (much smaller than $\mathcal{N}$ ).

The computational procedure can be split into two parts: an (expensive) offline part devoted to the computation of parameter independent terms and performed only once, and an (inexpensive) online part devoted to the computation of parameter dependent terms and performed many times. We report the two steps for the calculations and their computational costs in term of operations:

- offline: we compute $N$ FE solutions $u_{h}\left(\mu^{n}\right)$, one for each $\mu^{n} \in S_{N}$. We assemble and store the parameter-independent matrices $\underline{A}_{N}^{q}=\underline{Z}^{T} \underline{A}_{h}^{q} \underline{Z}$ for $q=1, \ldots, Q$ and the same is done for $\underline{F}_{N}=\underline{Z}^{T} \underline{F}_{h}$;

- online: we compute parameter-dependent functions $\sigma^{q}(\mu)$, the matrix $\underline{A}_{N}(\mu)=\sum_{q=1}^{Q} \sigma^{q}(\mu) \underline{A}_{N}^{q}$ is assembled, then the system $\underline{A}_{N}(\mu) \underline{u}_{N}(\mu)=\underline{F}_{N}$ is solved and we compute the output of interest $s_{N}(\mu)=\underline{F}_{N}^{T} \underline{u}_{N}(\mu)$.

All the online operations are independent of the dimension $\mathcal{N}$, and they depend only on $N$ and $Q$; since $N$ is normally really small, the output can be computed in real time, once all the offline calculations are carried out and the corresponding results are stored. For all the new values of $\mu$ we need $\mathcal{O}\left(Q N^{2}\right)$ assembling operations and $\mathcal{O}\left(\frac{2}{3} N^{3}\right)$ operations to solve the linear system. 
Other important aspects of the RB formulation are (i) the availability of an error bound theory to develop a rapid a posteriori error estimation based on residual calculations, see for example [25, 28], and (ii) the studies on a-priori convergence and error estimation, see $[16,24]$.

A crucial aspect is the availability of a good algorithm to select snapshots and basis functions to build the reduced basis approximation space. Given the higher powers of $N$ that appear in the online complexity estimates, it is crucial to control $N$ more tightly. We first construct, offline, an approximation that, over most of the domain, exhibits an error $\epsilon_{N}(\mu)=\|e(\mu)\|_{X}=\left\|u_{h}(\mu)-u_{N}(\mu)\right\|_{X}$ less than $\epsilon_{d}^{\text {prior }}$ : we begin with a first point $\mu^{1}\left(S_{N^{\prime}=1}=\left\{\mu^{1}\right\}\right)$; we next evaluate $\epsilon_{N^{\prime}=1}(\mu)$ over a large test sample of parameter points in $\mathcal{D}^{\mu}, \Sigma^{\text {prior }}$; we then choose for $\mu^{2}$ (and hence $S_{N^{\prime}=2}=\left\{\mu^{1}, \mu^{2}\right\}$ ) the maximizer of $\epsilon_{N^{\prime}=1}(\mu)$ over $\Sigma^{\text {prior }}$. We repeat this process until the maximum of $\epsilon_{N^{\prime}=N_{\text {prior }}}(\mu)$ over $\Sigma^{\text {prior }}$ is less than $\epsilon_{d}^{\text {prior }}$. Then, online, given a new value of the parameter, $\mu$, and an error tolerance $\epsilon_{d}^{\text {post }}(\mu)$, we essentially repeat this adaptive process - but now our sample points are drawn from $S_{N^{\text {prior }}}$, and the test sample is a singleton - $\mu$. Typically we choose $\epsilon_{d}^{\text {prior }} \ll \epsilon_{d}^{\text {post }}(\mu)$ since our test is not exhaustive; and therefore, typically, $N^{\text {post }}(\mu) \ll N^{\text {prior }}$. With the adaptive process we get higher accuracy at lower $N$ : modest reductions in $N$ can translate into measurable performance improvements. This procedure is very important not only to get a computationally cheaper and faster procedure but also to avoid ill-conditioning in matrix assembling procedures [30]. For this reason many different algorithms have been developed to apply also a posteriori error bounds adaptively, see [34, 24].

Important extensions on the application of RB methods in linear elasticity may involve a dual problem to treat general outputs [33, 19] (the latter can be either linear or quadratic [12]) as well as the treatment of more general operators [18] accounting also for inertial effects.

\section{Linear elasticity equations and their parametrization}

We briefly recall the elliptic equations for a general linear elasticity equilibrium problem $[15,2,5,32,35]$, considering a homogeneous body in a domain $\hat{\Omega} \in \mathbb{R}^{d}$ with boundary $\hat{\Gamma}$

$$
\frac{\partial \hat{\sigma}_{i j}}{\partial \hat{x}_{j}}+\hat{b}_{i}=0 \quad \text { in } \hat{\Omega}, 1 \leq i, j \leq d
$$

and boundary conditions on $\hat{\Gamma}$. Here $\hat{\sigma}_{i j}=\hat{C}_{i j k l} \hat{\varepsilon}_{k l}$ represent stresses, $\hat{b}_{i}$ the components of a given force field, $\hat{C}_{i j k l}$ is the elastic tensor ${ }^{1}$ (everywhere in this paper summation on repeated indices is understood). Thanks to the symmetry of the latter we can write

$$
\hat{\sigma}_{i j}=\hat{C}_{i j k l} \frac{\partial \hat{u}_{k}}{\partial \hat{x}_{l}}, 1 \leq i, j, k, l \leq d
$$

\footnotetext{
${ }^{1}$ If we consider isotropic materials the elastic tensor is given by $\hat{C}_{i j k l}=c_{1} \delta_{i j} \delta_{k l}+c_{2}\left(\delta_{i k} \delta_{j l}+\delta_{i l} \delta_{j k}\right)$, where $c_{1}$ and $c_{2}$ are the Lamé constants given by $c_{1}=\frac{E \nu}{(1+\nu)(1-2 \nu)}$ and $c_{2}=\frac{E}{2(1+\nu)}$, where $E$ is the Young Modulus and and $\nu$ is the Poisson coefficient. Thanks to the symmetry of $\hat{\sigma}_{i j}$ and $\hat{\varepsilon}_{k l}$, for the elastic tensor we have $\hat{C}_{i j k l}=\hat{C}_{j i k l}=\hat{C}_{i j l k}=\hat{C}_{k l i j}$. This formulation is still valid in the plane stress approximation, while when we consider orthotropic materials the elastic tensor should be redefined.
} 
where $\hat{u}_{k}$ represents a displacement. The linearized deformations $\hat{\varepsilon}_{k l}$ are given by

$$
\hat{\varepsilon}_{k l}=\frac{1}{2}\left(\frac{\partial \hat{u}_{k}}{\partial \hat{x}_{l}}+\frac{\partial \hat{u}_{l}}{\partial \hat{x}_{k}}\right), 1 \leq k, l \leq d,
$$

which represents the linearized strain tensor. For the problem (22) we consider Dirichlet conditions (where we impose a displacement $\hat{u}_{i}$, typically zero) on $\hat{\Gamma}_{D} \subset \hat{\Gamma}$. Precisely:

$$
\hat{u}=0, \quad \text { on } \hat{\Gamma}_{\mathrm{D}},
$$

and Neumann conditions (as we impose a distributed load or a free-stress condition) on $\hat{\Gamma} \backslash \hat{\Gamma}_{D}$ :

$$
\begin{gathered}
\hat{\sigma}_{i j} \hat{\mathrm{e}}_{j}^{n}=\hat{f}_{n} \hat{\mathrm{e}}_{i}^{n} \quad \text { on } \hat{\Gamma}_{\mathrm{N}}^{n}, \\
\hat{\sigma}_{i j} \hat{\mathrm{e}}_{j}^{n}=\hat{f}_{t} \hat{\mathrm{e}}_{i}^{t} \quad \text { on } \hat{\Gamma}_{\mathrm{N}}^{t},
\end{gathered}
$$

where $\hat{\mathrm{e}}_{i}^{n}$ and $\hat{\mathrm{e}}_{i}^{t}$ represent the components of normal $(n)$ and tangential $(t)$ unit vector, respectively. Note that $\hat{\Gamma}_{D} \cap \hat{\Gamma}_{N} \equiv 0$.

We now derive the weak formulation of (22), we introduce the functional space

$$
\hat{Y}=\left\{\hat{v} \in\left(H^{1}(\hat{\Omega})\right)^{d} \mid \hat{v}=0 \text { on } \hat{\Gamma}_{\mathrm{D}}\right\}
$$

and the associated seminorm in $\left(H^{1}(\hat{\Omega})\right)^{d}$ :

$$
|\hat{v}|_{\hat{Y}}=\left(\sum_{i=1}^{d}\left|\hat{v}_{i}\right|_{H^{1}(\hat{\Omega})}^{2}\right)^{1 / 2}=\left(\int_{\hat{\Omega}} \sum_{i, j=1}^{d}\left(\frac{\partial \hat{v}_{i}}{\partial \hat{x}_{j}}\right)^{2} d \hat{\Omega}\right)^{1 / 2}
$$

Multiplying (22) for the test function $\hat{v} \in \hat{Y}$, integrating on $\hat{\Omega}$, using the divergence theorem and applying boundary conditions we get

$$
\int_{\hat{\Omega}} \frac{\partial \hat{v}_{i}}{\partial \hat{x}_{j}} \hat{\sigma}_{i j} d \hat{\Omega}=\int_{\hat{\Omega}} \hat{v}_{i} \hat{b}_{i} d \hat{\Omega}+\int_{\hat{\Gamma}_{\mathrm{N}}} \hat{v}_{i} \hat{f}_{n} \hat{\mathrm{e}}_{i}^{n} d \hat{\Gamma}+\int_{\hat{\Gamma}_{\mathrm{N}}} \hat{v}_{i} \hat{f}_{t} \hat{\mathrm{e}}_{i}^{t} d \hat{\Gamma}
$$

In compact notation we can write

$$
\langle\hat{\mathcal{A}} \hat{u}, \hat{v}\rangle=\langle\hat{F}, \hat{v}\rangle, \quad \forall \hat{v} \in \hat{Y},
$$

where

$$
\begin{aligned}
\langle\hat{\mathcal{A}} \hat{w}, \hat{v}\rangle & =\int_{\hat{\Omega}} \frac{\partial \hat{v}_{i}}{\partial \hat{x}_{j}} \hat{C}_{i j k l} \frac{\partial \hat{w}_{k}}{\partial \hat{x}_{l}} d \hat{\Omega} \\
\langle\hat{F}, \hat{v}\rangle & =\left\langle\hat{F}_{f}, \hat{v}\right\rangle+\left\langle\hat{F}_{b}, \hat{v}\right\rangle
\end{aligned}
$$

with

$$
\left\langle\hat{F}_{f}, \hat{v}\right\rangle=\int_{\hat{\Gamma}_{\mathrm{N}}} \hat{v}_{i} \hat{f}_{n} \hat{\mathrm{e}}_{i}^{n} d \hat{\Gamma}+\int_{\hat{\Gamma}_{\mathrm{N}}} \hat{v}_{i} \hat{f}_{t} \hat{\mathrm{e}}_{i}^{t} d \hat{\Gamma}, \quad\left\langle\hat{F}_{b}, \hat{v}\right\rangle=\int_{\hat{\Omega}} \hat{v}_{i} \hat{b}_{i} d \hat{\Omega}
$$


In the case $\hat{\Omega}$ is made up by different portions of different materials, for example $R$ homogeneous subdomains $\hat{\Omega}^{r}$ such that

$$
\overline{\hat{\Omega}}=\bigcup_{r=1}^{R} \overline{\hat{\Omega}}^{r}
$$

where we indicate with $\bar{\Omega}$ the closure of $\hat{\Omega}$, the weak formulation is still governed by (30), however now we have

$$
\begin{aligned}
\langle\hat{\mathcal{A}} \hat{w}, \hat{v}\rangle & =\sum_{r=1}^{R} \int_{\hat{\Omega}^{r}} \frac{\partial \hat{v}_{i}}{\partial \hat{x}_{j}} \hat{C}_{i j k l}^{r} \frac{\partial \hat{w}_{k}}{\partial \hat{x}_{l}} d \hat{\Omega} \\
\langle\hat{F}, \hat{v}\rangle & =\left\langle\hat{F}_{f}, \hat{v}\right\rangle+\left\langle\hat{F}_{b}, \hat{v}\right\rangle
\end{aligned}
$$

with

$$
\left\langle\hat{F}_{b}, \hat{v}\right\rangle=\sum_{r=1}^{R} \int_{\hat{\Omega}^{r}} \hat{v}_{i} \hat{b}_{i}^{r} d \hat{\Omega}, \quad\left\langle\hat{F}_{f}, \hat{v}\right\rangle=\sum_{r=1}^{R}\left(\int_{\partial \hat{\Omega}^{r} \cap \hat{\Gamma}_{\mathrm{N}}} \hat{v}_{i} \hat{f}_{n}^{r} \hat{\mathrm{e}}_{i}^{n} d \hat{\Gamma}+\int_{\partial \hat{\Omega}^{r} \cap \hat{\Gamma}_{\mathrm{N}}} \hat{v}_{i} \hat{f}_{t}^{r} \hat{\mathrm{e}}_{i}^{t} d \hat{\Gamma}\right),
$$

$\hat{C}_{i j k l}^{r}$ is the elastic tensor in $\hat{\Omega}^{r}$, and $\left(\partial \hat{\Omega}^{r} \cap \hat{\Gamma}_{\mathrm{N}}\right)$ is the portion of $\hat{\Gamma}_{\mathrm{N}} \subset \overline{\hat{\Omega}}^{r}$.

\subsection{Parametrized formulation and mappings}

On problem (30) - (35) - (37) we highlight the parameters that are associated with variation in the geometry (domains), boundary conditions (loads), physical properties (materials) to apply the affine decomposition of the operators into different components.

In the case of geometrical parametrization, at each subdomain $\hat{\Omega}^{r}, r=1, \ldots, R$, a reference subdomain $\Omega^{r}, r=1, \ldots, R$, is associated so that $\bar{\Omega}=\bigcup_{r=1}^{R} \bar{\Omega}^{r}$ where, for each $\underline{\hat{x}} \in \hat{\Omega}^{r}$, $r=1, \ldots, R$, its corresponding image $\underline{x} \in \Omega^{r}$ is given by

$$
\underline{x}=\mathcal{G}^{r}(\mu ; \underline{\hat{x}})=\underline{G}^{r}(\mu) \underline{\hat{x}}+\underline{g}^{r}(\mu),
$$

and we get the following transformation to be applied to each derivative term

$$
\frac{\partial}{\partial \hat{x}_{i}}=\frac{\partial x_{j}}{\partial \hat{x}_{i}} \frac{\partial}{\partial x_{j}}=G_{j i}(\mu) \frac{\partial}{\partial x_{j}} .
$$

We introduce a new functional space $Y(\Omega)=\hat{Y}\left(\mathcal{G}^{-1}(\mu ; \Omega)\right)=\hat{Y}(\hat{\Omega})$, i.e.

$$
Y(\Omega)=\left\{v \in\left(H^{1}(\Omega)\right)^{d} \mid v=0 \text { on } \Gamma_{\mathrm{D}}\right\}
$$

and for all $\hat{w} \in \hat{Y}$, we define $w \in Y$ such that $w(\underline{x})=\hat{w}\left(\mathcal{G}^{-1}(\mu ; \underline{x})\right)$. We get

$$
\begin{aligned}
d \hat{\Omega} & =\operatorname{det} \underline{G}^{-1}(\mu) d \Omega, \\
d \hat{\Gamma} & =\left|\underline{G}^{-1}(\mu) \underline{\mathrm{e}}^{t}\right| d \Gamma,
\end{aligned}
$$


where $\underline{\mathrm{e}}^{t}$ is the tangential unit vector to $\Gamma$ and

$$
\left.\left|\underline{G}^{-1}(\mu) \underline{\mathrm{e}}^{t}\right|=\left(\sum_{i=1}^{d}\left(G_{i j} \mathrm{e}\right)_{j}^{t}\right)^{2}\right)^{1 / 2} .
$$

It follows that $\langle\mathcal{A}(\mu) w, v\rangle=\langle\hat{\mathcal{A}} \hat{w}, \hat{v}\rangle$ where $\hat{\mathcal{A}}$ is given by $(35)$ while $\mathcal{A}(\mu)$ by

$$
\begin{array}{r}
\langle\mathcal{A}(\mu) w, v\rangle=\sum_{r=1}^{R} \int_{\Omega^{r}}\left(G_{j j^{\prime}}^{r}(\mu) \frac{\partial w_{i}}{\partial x_{j}}\right) \hat{C}_{i j^{\prime} k l^{\prime}}^{r}(\mu)\left(G_{l l^{\prime}}^{r}(\mu) \frac{\partial v_{k}}{\partial x_{l}}\right) \operatorname{det}\left(\underline{G}^{r}(\mu)\right)^{-1} d \Omega \\
=\sum_{r=1}^{R} \int_{\Omega^{r}} \frac{\partial w_{i}}{\partial x_{j}}\left(G_{j j^{\prime}}^{r}(\mu) \hat{C}_{i j^{\prime} k l^{\prime}}^{r}(\mu) G_{l l^{\prime}}^{r}(\mu) \operatorname{det}(\underline{G}(\mu))^{-1}\right) \frac{\partial v_{k}}{\partial x_{l}} d \Omega \forall w, v \in Y .
\end{array}
$$

Note that we may introduce a parametric dependence directly in $\hat{C}_{i j k l}^{r}(\mu)$ when we consider physical parameters, not related with a geometrical transformation. In the same way: $\langle F(\mu) w, v\rangle=\langle\hat{F} \hat{w}, \hat{v}\rangle$ where $\hat{F}$ is given by (36) and

$$
\begin{aligned}
\langle F(\mu), v\rangle=\sum_{r=1}^{R}\left(\int_{\Omega^{r}}\left(\hat{b}_{i}^{r} \operatorname{det}\left(\underline{G}^{r}(\mu)\right)^{-1}\right)\right. & v_{i} d \Omega+\int_{\partial \Omega^{r} \cap \Gamma_{\mathrm{N}}}\left(\hat{f}_{n}^{r} \hat{\mathrm{e}}_{i}^{n}\left|\left(\underline{G}^{r}(\mu)\right)^{-1} \underline{\mathrm{e}}^{t}\right|\right) v_{i} d \Gamma \\
& \left.+\int_{\partial \Omega^{r} \cap \Gamma_{\mathrm{N}}}\left(\hat{f}_{t}^{r} \hat{\mathrm{e}}_{i}^{t}\left|\left(\underline{G}^{r}(\mu)\right)^{-1} \underline{\mathrm{e}}^{t}\right|\right) v_{i} d \Gamma\right) .
\end{aligned}
$$

The abstract formulation (30) is the following:

$$
\begin{aligned}
\langle\mathcal{A}(\mu) w, v\rangle & =\sum_{r=1}^{R} \int_{\Omega^{r}} \frac{\partial w_{i}}{\partial x_{j}} C_{i j k l}^{r}(\mu) \frac{\partial v_{k}}{\partial x_{l}} d \Omega \quad \forall w, v \in Y, \\
\langle F(\mu), v\rangle & =\left(\left\langle F_{b}(\mu), v\right\rangle+\left\langle F_{f}(\mu), v\right\rangle\right),
\end{aligned}
$$

where

$$
\begin{aligned}
\left\langle F_{b}(\mu), v\right\rangle & =\sum_{r=1}^{R} \int_{\Omega^{r}} b_{i}^{r}(\mu) v_{i} d \Omega, \\
\left\langle F_{f}(\mu), v\right\rangle & =\sum_{r=1}^{R}\left(\int_{\partial \Omega^{r} \cap \Gamma_{\mathrm{N}}} f_{i}^{n r}(\mu) v_{i} d \Gamma+\int_{\partial \Omega^{r} \cap \Gamma_{\mathrm{N}}} f_{i}^{t r}(\mu) v_{i} d \Gamma\right) .
\end{aligned}
$$

The compact tensor $C_{i j k l}^{r}(\mu)$ is given by

$$
C_{i j k l}^{r}(\mu)=G_{j j^{\prime}}^{r}(\mu) \hat{C}_{i j^{\prime} k l^{\prime}}^{r}(\mu) G_{l l^{\prime}}^{r}(\mu) \operatorname{det}\left(\underline{G}^{r}(\mu)\right)^{-1},
$$

while $b_{i}^{r}(\mu), f_{i}^{n r}(\mu)$ and $f_{i}^{t r}(\mu)$ by

$$
\begin{aligned}
b_{i}^{r}(\mu) & =\hat{b}_{i}^{r} \operatorname{det}\left(\underline{G}^{r}(\mu)\right)^{-1}, \\
f_{i}^{n r}(\mu) & =\hat{f}_{n}^{r} \hat{\mathrm{e}}_{i}^{n}\left|\left(\underline{G}^{r}(\mu)\right)^{-1} \underline{\mathrm{e}}^{t}\right|, \\
f_{i}^{t r}(\mu) & =\hat{f}_{t}^{r} \hat{\mathrm{e}}_{i}^{t}\left|\left(\underline{G}^{r}(\mu)\right)^{-1} \underline{\mathrm{e}}^{t}\right| .
\end{aligned}
$$


The terms arising from the affine decomposition are:

$$
\Theta^{q(i, j, k, l, r)}(\mu)=C_{i j k l}^{r}(\mu), \quad\left\langle\mathcal{A}^{q(i, j, k, l, r)} w, v\right\rangle=\int_{\Omega} \frac{\partial v_{i}}{\partial x_{j}} \frac{\partial w_{k}}{\partial x_{l}}
$$

and $q:\{1, \ldots, d\}^{4} \times\{1, \ldots, R\} \rightarrow\left\{1, \ldots, Q_{\mathcal{A}}\right\}$.

Thanks to the symmetry of $C_{i j k l}(\mu), Q_{\mathcal{A}}$ can be assumed as $d^{2}\left(d^{2}+1\right) R / 2$.

\subsection{Helmholtz equations}

To account also for inertial effects due to harmonic loads with frequency $\omega$, the D'Alembert principle leads to the following equilibrium equations

$$
\frac{\partial \hat{\Sigma}_{i j}}{\partial \hat{x}_{j}}+\hat{B}_{i}=\rho \frac{\partial^{2} \hat{U}_{i}}{\partial \hat{t}^{2}} \quad \text { in } \hat{\Omega}, \quad \forall i=1, . ., d,
$$

where $\hat{\Sigma}_{i j}=\hat{\sigma}_{i j} e^{-\kappa \omega t}$ (with $\kappa$ imaginary unit) are the harmonic stresses, $\hat{B}_{i}=\hat{b}_{i} e^{-\kappa \omega t}$ the harmonic volume forces, $\hat{U}_{i}=\hat{u}_{i} e^{-\kappa \omega t}$ the harmonic response of the system and $\rho$ the material density. The same boundary conditions than in the steady case apply. From (56), we get

$$
\frac{\partial \hat{\sigma}_{i j}}{\partial \hat{x}_{j}}+\hat{b}_{i}+\rho \omega^{2} \hat{u}_{i}=0 \quad \text { in } \hat{\Omega}
$$

so that $\hat{\sigma}_{i j}, \hat{b}_{i}$ and $\hat{u}_{i}$ (functions of $\omega$ ) represent the amplitude of the same quantities as in the steady case. The weak formulation of (57) is

$$
\langle\hat{\mathcal{A}} \hat{w}, \hat{v}\rangle=\int_{\hat{\Omega}} \hat{v}_{i} \hat{b}_{i} d \hat{\Omega}+\int_{\hat{\Gamma}_{\mathrm{N}}} \hat{v}_{i} \hat{f}_{n} \hat{\mathrm{e}}_{i}^{n} d \hat{\Gamma}+\int_{\hat{\Gamma}_{\mathrm{N}}} \hat{v}_{i} \hat{f}_{t} \hat{\mathrm{e}}_{i}^{t} d \hat{\Gamma}
$$

where

$$
\langle\hat{\mathcal{A}} \hat{w}, \hat{v}\rangle=\int_{\hat{\Omega}}\left(\frac{\partial \hat{v}_{i}}{\partial \hat{x}_{j}} \hat{C}_{i j k l} \frac{\partial \hat{w}_{k}}{\partial \hat{x}_{l}}-\rho \omega^{2} \hat{w}_{i} \hat{v}_{i}\right) d \hat{\Omega} .
$$

\subsection{Examples of parametrization}

We consider various kind of parametrizations, which characterize:

(a) geometry (domain);

(b) engineering: load intensity and direction;

(c) physics (material properties): Young modulus (E), shear modulus (G) and Poisson coefficient $(\nu)$

(d) multi subdomains for modular structures;

Applications will concern isotropic constitutive law (where $\hat{C}_{i j k l}$ depends on $E, G$ and $\nu$ ), orthotropic materials (where $\hat{C}_{i j k l}$ depends on $E_{x}, E_{y}, \nu_{x y}, \nu_{y x}$ and $G_{x y}$ ), general orthotropic law (where $\hat{C}_{i j k l}$ depends on $E_{x}, E_{y}, \nu_{x y}, \nu_{y x}, G_{x y}$ and also on the fibers angle $\theta$ with respect to the load direction). 


\section{Isotropic materials in plane strain}

We propose a first application concerning an isotropic material in plane strain formulation. We compare the solutions obtained by the reduced basis method and by the finite element method, by computing the errors on the $H^{1}$ semi-norm and we provide some indications on computational times and savings.

The plane strain approximation is used to study linear elasticity problems in two-dimensional domains $[13,6]$, considering a generic section and assuming equal to zero the deformations in the orthogonal direction of the section. Starting from (23) we can write

$$
\left\{\begin{array}{c}
\hat{\sigma}_{x} \\
\hat{\sigma}_{y} \\
\hat{\sigma}_{z} \\
\hat{\tau}_{x y} \\
\hat{\tau}_{x z} \\
\hat{\tau}_{y z}
\end{array}\right\}=\frac{E}{(1+\nu)(1-2 \nu)}\left[\begin{array}{cccccc}
1-\nu & \nu & \nu & 0 & 0 & 0 \\
\nu & 1-\nu & \nu & 0 & 0 & 0 \\
\nu & \nu & 1-\nu & 0 & 0 & 0 \\
0 & 0 & 0 & \frac{1-2 \nu}{2} & 0 & 0 \\
0 & 0 & 0 & 0 & \frac{1-2 \nu}{2} & 0 \\
0 & 0 & 0 & 0 & 0 & \frac{1-2 \nu}{2}
\end{array}\right]\left\{\begin{array}{c}
\hat{\varepsilon}_{x} \\
\hat{\varepsilon}_{y} \\
\hat{\varepsilon}_{z} \\
\hat{\gamma}_{x y} \\
\hat{\gamma}_{x z} \\
\hat{\gamma}_{y z}
\end{array}\right\},
$$

where $\hat{\gamma}_{i j}=2 \hat{\varepsilon}_{i j}$. We impose the plane strain approximation:

$$
\hat{\varepsilon}_{z}=\hat{\gamma}_{x z}=\hat{\gamma}_{y z}=0
$$

where $z$ is the direction orthogonal to the section $x, y$, so that from (60) we get the plane strain formulation:

$$
\left\{\begin{array}{c}
\hat{\sigma}_{x} \\
\hat{\sigma}_{y} \\
\hat{\tau}_{x y}
\end{array}\right\}=\frac{E}{(1+\nu)(1-2 \nu)}\left[\begin{array}{ccc}
1-\nu & \nu & 0 \\
\nu & 1-\nu & 0 \\
0 & 0 & \frac{1-2 \nu}{2}
\end{array}\right]\left\{\begin{array}{c}
\hat{\varepsilon}_{x} \\
\hat{\varepsilon}_{y} \\
\hat{\gamma}_{x y}
\end{array}\right\} .
$$

In this case the Lamé constants are given by

$$
\begin{gathered}
c_{1}=\lambda=\frac{E \nu}{(1+\nu)(1-2 \nu)}, \\
c_{2}=G=\frac{E}{2(1+\nu)},
\end{gathered}
$$

where $G$ is the elastic tangential modulus for the material.

\subsection{A Bi-material rod}

We consider a long and isotropic structure with a compression load on one side of its section (the other one is clamped). See Figure 2. The parameters we are considering are (Figure 3 (left)): 


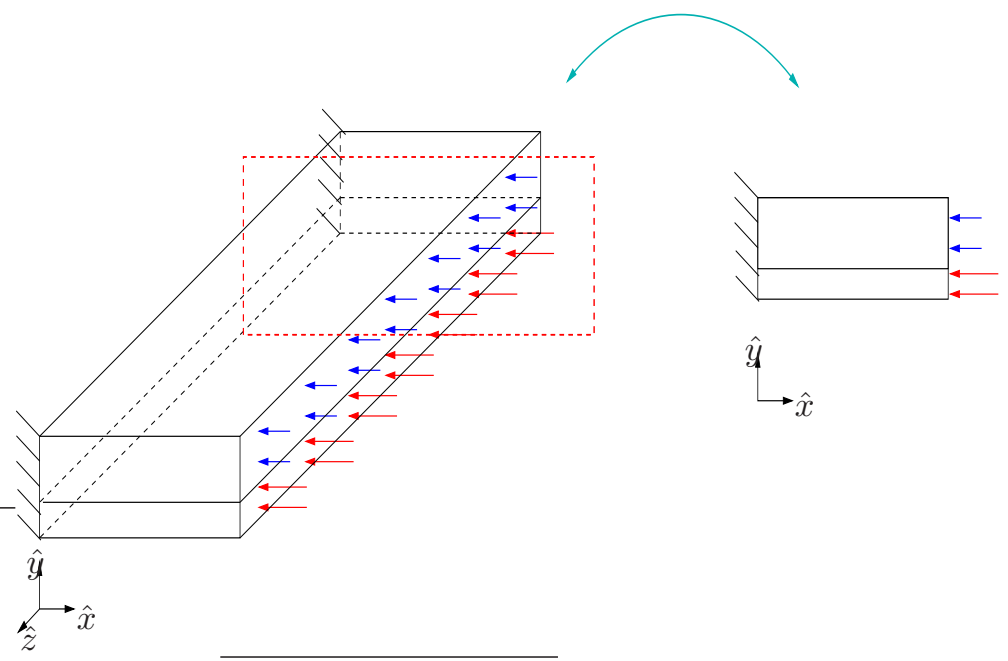

Figure 2: Bi-material long and isotropic structure with a compression load in plane strain formulation.
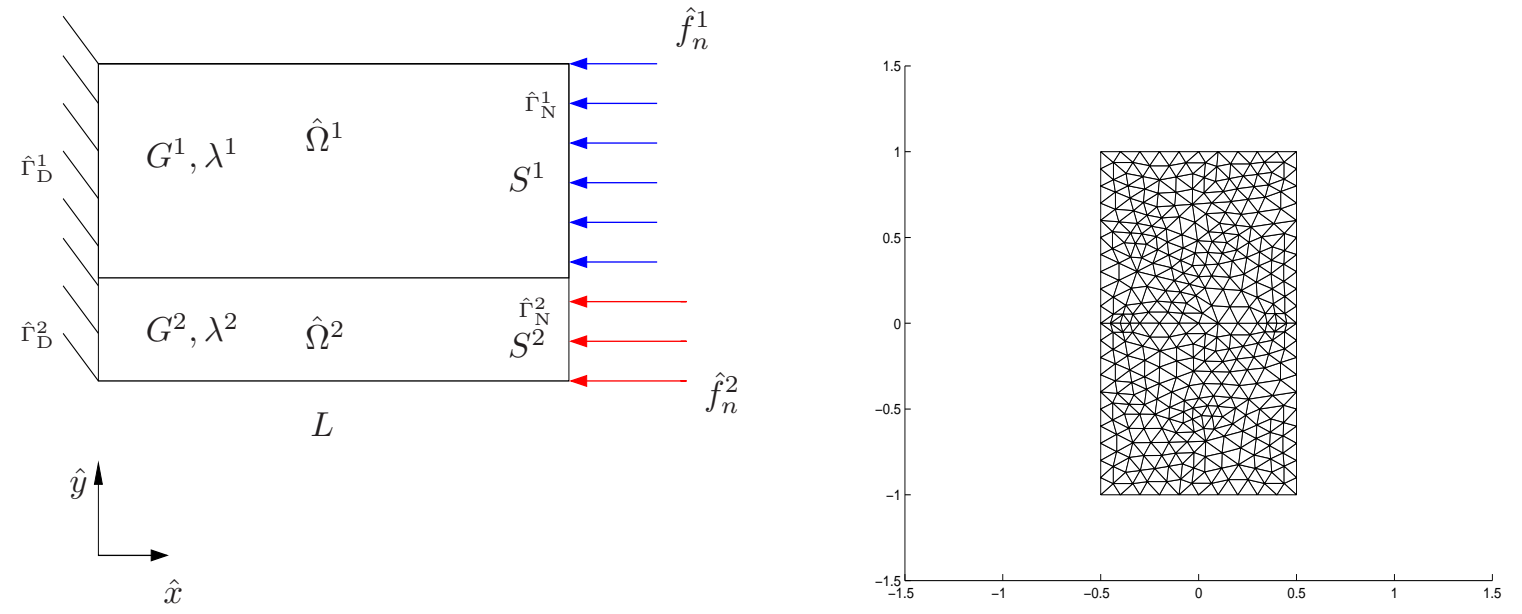

Figure 3: Schematic representation of the rod (left); reference mesh (right) made up by 680 elements.

- Geometry: the domain $\hat{\Omega}$ is made up by two variable subdomains: $\hat{\Omega}^{1}=\left(L, S^{1}\right)$ and $\hat{\Omega}^{2}=\left(L, S^{2}\right)$, with $L, S^{1}$ and $S^{2}$ being geometrical parameters.

- Physics: the materials in the subdomains are parametrized by the Lamé coefficients, i.e. $G^{1}, G^{2}, \lambda^{1}$ and $\lambda^{2}$, bound to Young modulus and Poisson coefficient by (63) and (64).

- Engineering: the loads $\hat{f}_{n}^{1}=-\frac{P}{S^{1}}$ and $\hat{f}_{n}^{2}=-\frac{P}{S^{2}}$ depend on a variable total force $P$ acting on each subdomain.

The vector of parameters is: $\mu=\left\{G^{1}, G^{2}, \lambda^{1}, \lambda^{2}, L, S^{1}, S^{2}, P\right\} \in \mathcal{D}^{\mu} \subset \mathbb{R}^{P=8}$. 
The boundary conditions on $\hat{\Gamma}_{\mathrm{D}}=\hat{\Gamma}_{\mathrm{D}}^{1} \cup \hat{\Gamma}_{\mathrm{D}}^{2}$ are:

$$
\hat{u}=0 \quad \text { on } \hat{\Gamma}_{\mathrm{D}} \text {, }
$$

while on $\hat{\Gamma}_{\mathrm{N}}=\hat{\Gamma}_{\mathrm{N}}^{1} \cup \hat{\Gamma}_{\mathrm{N}}^{2}$ we have:

$$
\hat{\sigma}_{i j} \hat{\mathrm{e}}_{j}^{n}= \begin{cases}\hat{f}_{n}^{r} \hat{\mathrm{e}}_{i}^{n} & \text { on } \hat{\Gamma}_{\mathrm{N}}^{r} \\ 0 & \text { on } \hat{\Gamma} \backslash\left(\hat{\Gamma}_{\mathrm{D}} \cup \hat{\Gamma}_{\mathrm{N}}\right)\end{cases}
$$

where $r=1,2$. Note that $\hat{f}_{t}$ is zero.

The mathematical formulation of the problem is: given $\mu \in \mathcal{D}^{\mu} \subset \mathbb{R}^{P=8}$, find $s(\mu)=$ $\langle\hat{L}, \hat{u}\rangle$, where $\hat{u} \in \hat{Y}=\left(H^{1}(\hat{\Omega})\right)^{2}$ is the solution of

$$
\langle\hat{\mathcal{A}} \hat{u}, \hat{v}\rangle=\langle\hat{F}, \hat{v}\rangle, \forall \hat{v} \in \hat{Y}
$$

and

$$
\begin{gathered}
\langle\hat{L}, \hat{v}\rangle=\langle\hat{F}, \hat{v}\rangle, \forall \hat{v} \in \hat{Y}, \\
\langle\hat{\mathcal{A}} \hat{w}, \hat{v}\rangle=\sum_{r=1}^{R} \int_{\hat{\Omega}^{r}} \frac{\partial \hat{v}_{i}}{\partial \hat{x}_{j}} \hat{C}_{i j k l}^{r} \frac{\partial \hat{w}_{k}}{\partial \hat{x}_{l}} d \hat{\Omega} \quad \forall \hat{w}, \hat{v} \in \hat{Y}, \\
\langle\hat{F}, \hat{v}\rangle=\sum_{r=1}^{R} \int_{\partial \hat{\Omega}^{r} \cap \hat{\Gamma}_{\mathrm{N}}} \hat{v}_{i} \hat{f}_{n}^{r} \hat{\mathrm{e}}_{i}^{n} d \hat{\Gamma} \quad \forall \hat{v} \in \hat{Y},
\end{gathered}
$$

where $R=2$.

The geometrical transformation to the reference domain is $\mathcal{G}^{r}(\hat{x})(\mu): \hat{\Omega} \longrightarrow \Omega$, given by (38), where $g=0$ and

$$
\underline{G}^{1}(\mu)=\left[\begin{array}{cc}
\frac{1}{L} & 0 \\
0 & \frac{1}{S^{1}}
\end{array}\right] \quad \underline{G}^{2}(\mu)=\left[\begin{array}{cc}
\frac{1}{L} & 0 \\
0 & \frac{1}{S^{2}}
\end{array}\right] .
$$

For $\Omega^{1}$ :

$$
\begin{aligned}
d \hat{\Omega} & =\operatorname{det} \underline{G}^{1-1}(\mu) d \Omega=L S^{1} d \Omega \\
d \hat{\Gamma} & =\left|\underline{G}^{1-1}(\mu) \underline{\mathrm{e}}^{t}\right| d \Gamma=S^{1} d \Gamma
\end{aligned}
$$

while for $\Omega^{2}$ :

$$
\begin{aligned}
d \hat{\Omega} & =\operatorname{det} \underline{G}^{2-1}(\mu) d \Omega=L S^{2} d \Omega \\
d \hat{\Gamma} & =\left|\underline{G}^{2-1}(\mu) \underline{\mathrm{e}}^{t}\right| d \Gamma=S^{2} d \Gamma .
\end{aligned}
$$

Introducing all the parameters in the problem and in the domain transformation we obtain:

$$
\begin{gathered}
\langle\mathcal{A}(\mu) w, v\rangle=\langle\hat{\mathcal{A}} \hat{w}, \hat{v}\rangle=\sum_{r=1}^{R} \int_{\Omega^{r}} \frac{\partial w_{i}}{\partial x_{j}} C_{i j k l}^{r}(\mu) \frac{\partial v_{k}}{\partial x_{l}} d \Omega \quad \forall w, v \in Y, \\
\langle F(\mu), v\rangle=\langle\hat{F}, \hat{v}\rangle=\sum_{r=1}^{R} \int_{\partial \Omega^{r} \cap \Gamma_{\mathrm{N}}} f_{i}^{n r}(\mu) v_{i} d \Gamma \quad \forall v \in Y,
\end{gathered}
$$


and (74) (compacting the notation so that $\frac{\partial w_{i}}{\partial x_{j}}=w_{i, j}$ ) becomes

$$
\begin{aligned}
\langle\mathcal{A}(\mu) w, v\rangle & =\frac{S^{1}}{L}\left(2 G^{1}+\lambda^{1}\right) \int_{\Omega^{1}} v_{1,1} w_{1,1} d \Omega+\lambda^{1} \int_{\Omega^{1}}\left(v_{2,2} w_{1,1}+v_{1,1} w_{2,2}\right) d \Omega+ \\
& +\frac{L}{S^{1}} G^{1} \int_{\Omega^{1}} v_{1,2} w_{1,2} d \Omega+G^{1} \int_{\Omega^{1}}\left(v_{1,2} w_{2,1}+v_{2,1} w_{1,2}\right) d \Omega+ \\
& +\frac{S^{1}}{L} G^{1} \int_{\Omega^{1}} v_{2,1} w_{2,1} d \Omega+\frac{L}{S^{1}}\left(2 G^{1}+\lambda^{1}\right) \int_{\Omega^{1}} v_{2,2} w_{2,2} d \Omega+ \\
& +\frac{S^{2}}{L}\left(2 G^{2}+\lambda^{2}\right) \int_{\Omega^{2}} v_{1,1} w_{1,1} d \Omega+\lambda^{2} \int_{\Omega^{2}}\left(v_{2,2} w_{1,1}+v_{1,1} w_{2,2}\right) d \Omega+ \\
& +\frac{L}{S^{2}} G^{2} \int_{\Omega^{2}} v_{1,2} w_{1,2} d \Omega+G^{2} \int_{\Omega^{2}}\left(v_{1,2} w_{2,1}+v_{2,1} w_{1,2}\right) d \Omega+ \\
& +\frac{S^{2}}{L} G^{2} \int_{\Omega^{2}} v_{2,1} w_{2,1} d \Omega+\frac{L}{S^{2}}\left(2 G^{2}+\lambda^{2}\right) \int_{\Omega^{1}} v_{2,2} w_{2,2} d \Omega
\end{aligned}
$$

By the affine decomposition we write:

$$
\begin{aligned}
\Theta^{1}(\mu) & =\frac{S^{1}}{L}\left(2 G^{1}+\lambda^{1}\right), & \left\langle\mathcal{A}^{1} w, v\right\rangle & =\int_{\Omega^{1}} v_{1,1} w_{1,1} d \Omega, \\
\Theta^{2}(\mu) & =\lambda^{1}, & \left\langle\mathcal{A}^{2} w, v\right\rangle & =\int_{\Omega^{1}}\left(v_{2,2} w_{1,1}+v_{1,1} w_{2,2}\right) d \Omega, \\
\Theta^{3}(\mu) & =\frac{L}{S^{1}} G^{1}, & \left\langle\mathcal{A}^{3} w, v\right\rangle & =\int_{\Omega^{1}} v_{1,2} w_{2,1} d \Omega, \\
\Theta^{4}(\mu) & =G^{1}, & \left\langle\mathcal{A}^{4} w, v\right\rangle & =\int_{\Omega^{1}}\left(v_{1,2} w_{2,1}+v_{2,1} w_{1,2}\right) d \Omega, \\
\Theta^{5}(\mu) & =\frac{S^{1}}{L} G^{1}, & \left\langle\mathcal{A}^{5} w, v\right\rangle & =\int_{\Omega^{1}} v_{2,1} w_{2,1} d \Omega, \\
\Theta^{6}(\mu) & =\frac{L}{S^{1}}\left(2 G^{1}+\lambda^{1}\right), & \left\langle\mathcal{A}^{6} w, v\right\rangle & =\int_{\Omega^{1}} v_{2,2} w_{2,2} d \Omega, \\
\Theta^{7}(\mu) & =\frac{S^{2}}{L}\left(2 G^{2}+\lambda^{2}\right), & \left\langle\mathcal{A}^{7} w, v\right\rangle & =\int_{\Omega^{2}} v_{1,1} w_{1,1} d \Omega, \\
\Theta^{8}(\mu) & =\lambda^{2}, & \left\langle\mathcal{A}^{8} w, v\right\rangle & =\int_{\Omega^{2}}\left(v_{2,2} w_{1,1}+v_{1,1} w_{2,2}\right) d \Omega,
\end{aligned}
$$




$$
\begin{array}{rlrl}
\Theta^{9}(\mu) & =\frac{L}{S^{2}} G^{2}, & \left\langle\mathcal{A}^{9} w, v\right\rangle=\int_{\Omega^{2}} v_{1,2} w_{2,1} d \Omega, \\
\Theta^{10}(\mu)=G^{2}, & \left\langle\mathcal{A}^{10} w, v\right\rangle=\int_{\Omega^{2}}\left(v_{1,2} w_{2,1}+v_{2,1} w_{1,2}\right) d \Omega, \\
\Theta^{11}(\mu)=\frac{S^{2}}{L} G^{2}, & \left\langle\mathcal{A}^{11} w, v\right\rangle=\int_{\Omega^{2}} v_{2,1} w_{2,1} d \Omega, \\
\Theta^{12}(\mu)=\frac{L}{S^{2}}\left(2 G^{2}+\lambda^{2}\right), & \left\langle\mathcal{A}^{12} w, v\right\rangle=\int_{\Omega^{2}} v_{2,2} w_{2,2} d \Omega .
\end{array}
$$

By using (50) and (53), (75) becomes

$$
\begin{aligned}
\langle F(\mu), v\rangle & =\int_{\partial \Omega^{1} \cap \Gamma_{\mathrm{N}}} f_{1}^{n 1}(\mu) v_{1} d \Gamma+\int_{\partial \Omega^{2} \cap \Gamma_{\mathrm{N}}} f_{1}^{n 2}(\mu) v_{1} d \Gamma= \\
& =-\int_{\partial \Omega^{1} \cap \Gamma_{\mathrm{N}}} \frac{P}{S^{1}} S^{1} v_{1} d \Gamma-\int_{\partial \Omega^{2} \cap \Gamma_{\mathrm{N}}} \frac{P}{S^{2}} S^{2} v_{1} d \Gamma= \\
& =-P\left(\int_{\partial \Omega^{1} \cap \Gamma_{\mathrm{N}}} v_{1} d \Gamma+\int_{\partial \Omega^{2} \cap \Gamma_{\mathrm{N}}} v_{1} d \Gamma\right)=-P \int_{\Gamma_{\mathrm{N}}} v_{1} d \Gamma .
\end{aligned}
$$

The output of interest is the average displacement on $\Gamma_{\mathrm{N}}^{n}$

$$
s(\mu)=-\int_{\Gamma_{\mathrm{N}}} u_{1}(\mu) d \Gamma \quad \text { for } \mu \in \mathcal{D}^{\mu} .
$$

\subsection{Numerical results}

We report as example in Figure 4 a visualization of displacements for the problem we are considering, assuming a bi-material section made up of aluminium and steel, for example see $[7]$.

The reduced basis method is very efficient for the solution of parametrized problems. We report the Matlab cputime using an implementation on Intel Pentium IV, $2.6 \mathrm{GHz}$ and 512 MB RAM. In Figure 5 the comparisons are carried out by computing 80 and $50 \mathrm{FE}$ and RB solutions for the same parameters combinations and for different dimensions of the RB space $(N)$. RB allows a computational saving of $95 \%$ with respect to $\mathrm{FE}$, without considering time to build (and rebuild) meshes using $\mathrm{FE}$ or to assemble the basis for $\mathrm{RB}$. The average time to get a FE solution is $1.4 s$ while to get a $\mathrm{RB}$ solution for $N=20$ is $0.06 s$.

We report now some results concerning the rapid convergence of the method: in Figure 6 we show the max and the average relative error $\left|e_{N}(\mu)\right|_{Y}=\frac{\left|u_{h}(\mu)-u_{N}(\mu)\right|_{Y}}{\left|u_{h}(\mu)\right|_{Y}}$ testing a large number of different configurations (500) depending on 8 parameters and varying $N$. The basis is optimized according to the procedure described in Section 3. The points (spots) for every $N$ represent the error for a single configuration. 

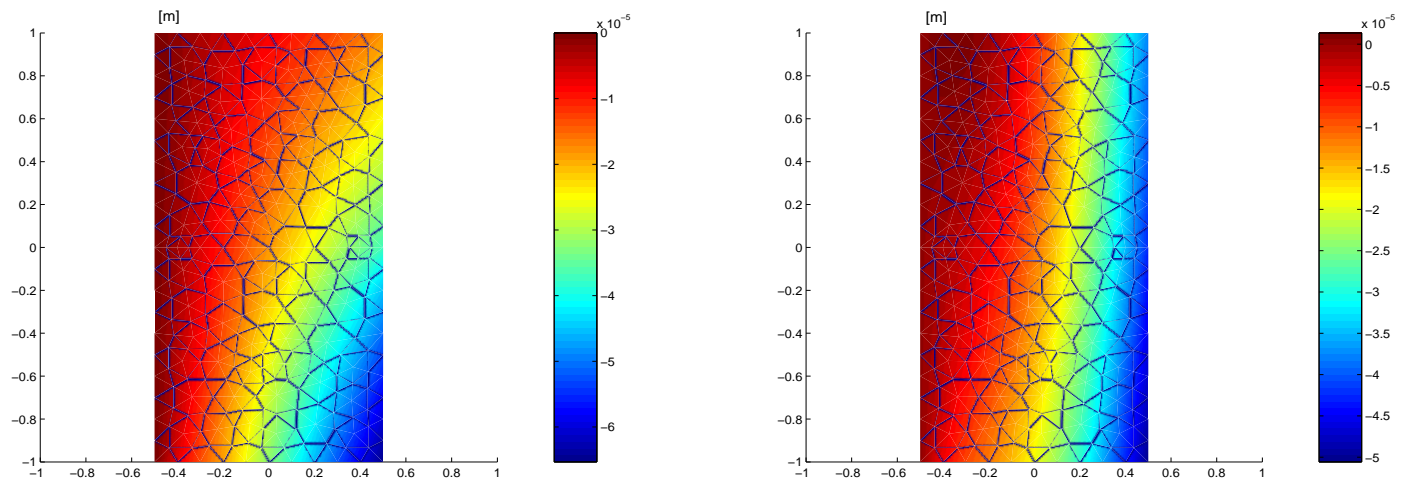

Figure 4: Displacements: $u$ in $x$ direction (left) and $v$ in $y$ direction (right) considering a bi-material section.
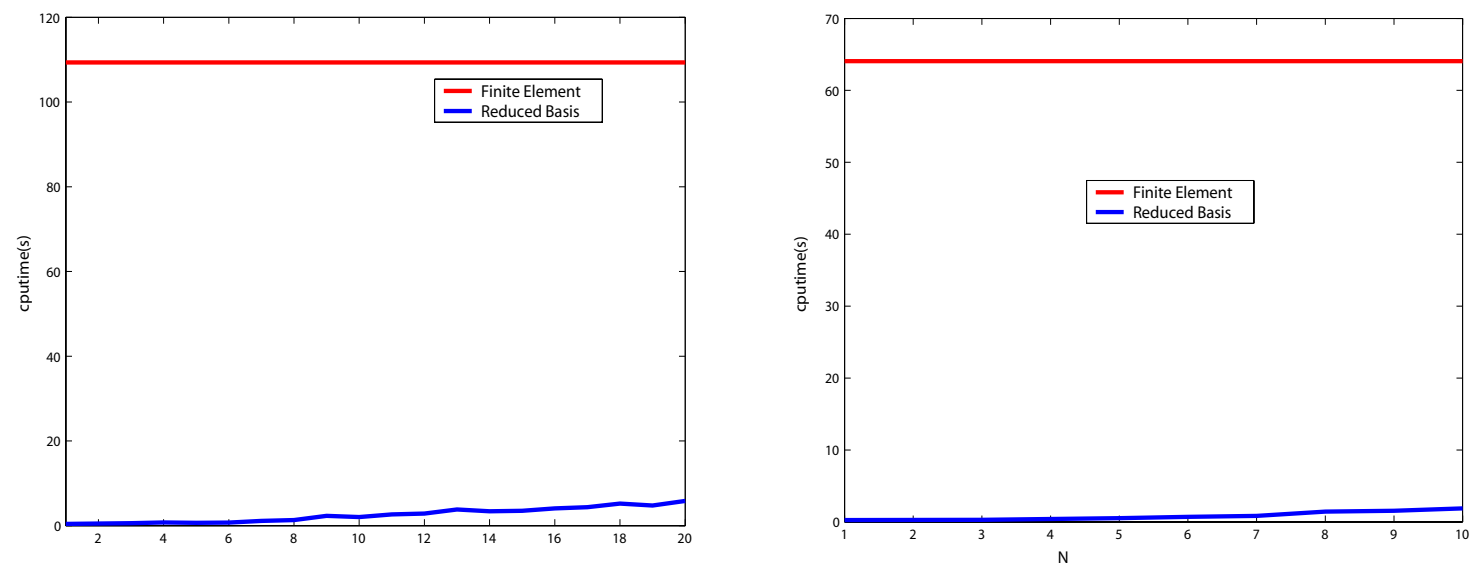

Figure 5: Comparison of computational times for 80 parametrized solutions (left) and 50 parametrized solutions (right) using finite element method and reduced basis method by increasing $N$.

\section{Applications to orthotropic materials in plane stress}

We consider an orthotropic material and one of its generalized model used to describe a composite material, made up of fibers and matrices, changing the fibers direction with respect to the load, using a micro-mechanical approach $[4,9]$. Composite materials are often designed to demonstrate some specific mechanical properties in the framework of material tayloring where it is important to get a real-time answer (i.e. output representing stress or displacement) when loads, mechanical or geometrical properties are varying. Composite materials are considered working in a regime of plane stress and in a two-dimensional lamina, the width $(z)$ is in fact considered very small compared with the planar dimension $(x, y)$. We make the following assumptions: 


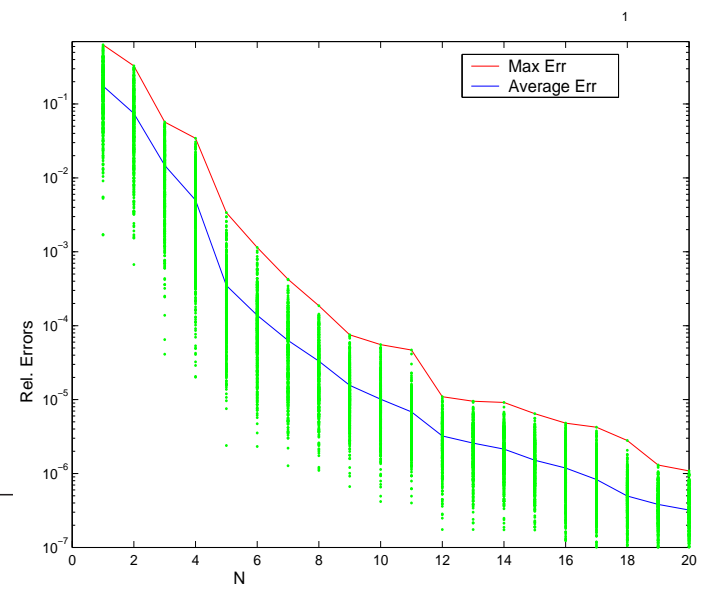

Figure 6: Max and average relative error testing a large number of different configurations (500) depending on 8 parameters and varying $N$.

- matrices have an elastic, linear, homogeneous and isotropic behavior;

- fibers have an elastic, linear, homogeneous and isotropic behavior and are aligned;

- the lamina should have an elastic, linear and orthotropic or general orthotropic behavior;

- there are no holes in the material and between fibers and matrices;

- we refer to a small volume of material on which we consider stresses and displacements as uniform.

A simple approach to get global mechanical properties of the lamina starting from the ones of fibers and matrices is provided by the Reuss-Voigt model [4] where

$$
\begin{array}{r}
E_{x}=c_{m} E_{m}+c_{f} E_{f}, E_{y}=\frac{E_{m} E_{f}}{c_{m} E_{f}+c_{f} E_{m}}, \\
\nu_{x y}=c_{m} \nu_{m}+c_{f} \nu_{f}, \nu_{y x}=\frac{E_{y}}{E_{x}} \nu_{x}, \\
G_{x y}=\frac{G_{m} G_{f}}{c_{m} G_{f}+c_{f} G_{m}},
\end{array}
$$

under the constraint of volumetric fractions composition:

$$
c_{m}+c_{f}=1 .
$$

Note that $G_{f}=\frac{E_{f}}{2\left(1+\nu_{f}\right)}$ and $G_{m}=\frac{E_{m}}{2\left(1+\nu_{m}\right)}$. The subscripts $f$ and $m$ represent fibers and matrices respectively. See [8] for more analytical and experimental considerations about 
composite materials homogenization. We report the mathematical formulation of the plane stress approximation [3] for an orthotropic material:

$$
\left\{\begin{array}{c}
\hat{\varepsilon}_{x} \\
\hat{\varepsilon}_{y} \\
\hat{\varepsilon}_{z} \\
\hat{\gamma}_{x y} \\
\hat{\gamma}_{x z} \\
\hat{\gamma}_{y z}
\end{array}\right\}=\left[\begin{array}{cccccc}
\frac{1}{E_{x}} & -\frac{\nu_{x y}}{E_{x}} & -\frac{\nu_{x z}}{E_{x}} & 0 & 0 & 0 \\
-\frac{\nu_{y x}}{E_{y}} & \frac{1}{E_{y}} & -\frac{\nu_{y z}}{E_{y}} & 0 & 0 & 0 \\
-\frac{\nu_{z x}}{E_{z}} & -\frac{\nu_{z y}}{E_{z}} & \frac{1}{E_{z}} & 0 & 0 & 0 \\
0 & 0 & 0 & \frac{1}{G_{x y}} & 0 & 0 \\
0 & 0 & 0 & 0 & \frac{1}{G_{x y}} & 0 \\
0 & 0 & 0 & 0 & 0 & \frac{1}{G_{x y}}
\end{array}\right]\left\{\begin{array}{c}
\hat{\sigma}_{x} \\
\hat{\sigma}_{y} \\
\hat{\sigma}_{z} \\
\hat{\tau}_{x y} \\
\hat{\tau}_{x z} \\
\hat{\tau}_{y z}
\end{array}\right\} .
$$

By definition we impose

$$
\hat{\sigma}_{z}=\hat{\tau}_{x z}=\hat{\tau}_{y z}=0,
$$

where $z$ is the direction along which the width is very small (and can be neglected). Substituting (84) in (83), we get

$$
\left\{\begin{array}{c}
\hat{\varepsilon}_{x} \\
\hat{\varepsilon}_{y} \\
\hat{\gamma}_{x y}
\end{array}\right\}=\left[\begin{array}{ccc}
\frac{1}{E_{x}} & -\frac{\nu_{x y}}{E_{x}} & 0 \\
-\frac{\nu_{y x}}{E_{y}} & \frac{1}{E_{y}} & 0 \\
0 & 0 & \frac{1}{G_{x y}}
\end{array}\right]\left\{\begin{array}{c}
\hat{\sigma}_{x} \\
\hat{\sigma}_{y} \\
\hat{\tau}_{x y}
\end{array}\right\} .
$$

By inverting the matrix in (85), we find:

$$
\left\{\begin{array}{c}
\hat{\sigma}_{x} \\
\hat{\sigma}_{y} \\
\hat{\tau}_{x y}
\end{array}\right\}=\left[\begin{array}{ccc}
\frac{E_{x}}{1-\nu_{x y} \nu_{y x}} & \frac{\nu_{x y} E_{y}}{1-\nu_{x y} \nu_{y x}} & 0 \\
\frac{\nu_{y x} E_{x}}{1-\nu_{y x} \nu_{x y}} & \frac{E_{y}}{1-\nu_{x y} \nu_{y x}} & 0 \\
0 & 0 & G_{x y}
\end{array}\right]\left\{\begin{array}{c}
\hat{\varepsilon}_{x} \\
\hat{\varepsilon}_{y} \\
\hat{\gamma}_{x y}
\end{array}\right\},
$$

which we denote by

$$
\{\hat{\sigma}\}=[Q]\{\hat{\varepsilon}\}
$$

The elements of the matrix $Q_{r s}$ represent the terms of the stiffness tensor $\hat{C}_{i j k l}$ of the isotropic case. The relationship between $Q_{r s}$ and the physical coefficients describing the material is

$$
\left\{\begin{array}{c}
\hat{\sigma}_{x} \\
\hat{\sigma}_{y} \\
\hat{\tau}_{x y}
\end{array}\right\}=\left[\begin{array}{ccc}
Q_{11} & Q_{12} & 0 \\
Q_{21} & Q_{22} & 0 \\
0 & 0 & Q_{66}
\end{array}\right]\left\{\begin{array}{c}
\hat{\varepsilon}_{x} \\
\hat{\varepsilon}_{y} \\
\hat{\gamma}_{x y}
\end{array}\right\}
$$


where

$$
\begin{aligned}
Q_{11} & =\frac{E_{x}}{1-\nu_{x y} \nu_{y x}}, \\
Q_{12} & =\frac{\nu_{x y} E_{y}}{1-\nu_{x y} \nu_{y x}}=\frac{\nu_{y x} E_{x}}{1-\nu_{y x} \nu_{x y}}=Q_{21}, \\
Q_{22} & =\frac{E_{y}}{1-\nu_{x y} \nu_{y x}}, \\
Q_{66} & =G_{x y} .
\end{aligned}
$$

Remark. In the parametric case the condition $Q_{r s}>0$ yields: $\nu_{x y}^{2} \leq \frac{E_{x}}{E_{y}}$. The conditions to guarantee that $Q$ is symmetric positive semi-definite are that $Q_{11} \geq 0, Q_{22} \geq 0$, and $Q_{66} \geq 0$ (i.e. $\nu_{x y}^{2} \leq \frac{E_{x}}{E_{y}}$ and $G_{x y}>0$ ) and $\left|Q_{12}\right| \leq \sqrt{Q_{11} \cdot Q_{22}}$ (i.e. $\nu_{x y}^{2} \leq \frac{E_{x}}{E_{y}}$ ). The (parametric) coercivity is guaranteed by the sufficient condition $Q_{12} \leq \frac{1}{2}\left(Q_{11}+Q_{22}\right)$ (i.e $\left.2 \nu_{x y}-1 \leq \frac{E_{x}}{E_{y}}\right)$. This last condition is related with the imposition of keeping the matrix diagonally dominant. See [14] and [1].

\subsection{An orthotropic lamina}

We first consider the case in which the fibers are aligned along the $x$ axis. There is a compression load applied to $\hat{\Gamma}_{\mathrm{N}}$ and the lamina is clamped on $\hat{\Gamma}_{\mathrm{D}}$; no loads on upper and lower side. We have the following parameters (Figure 7 (left)):
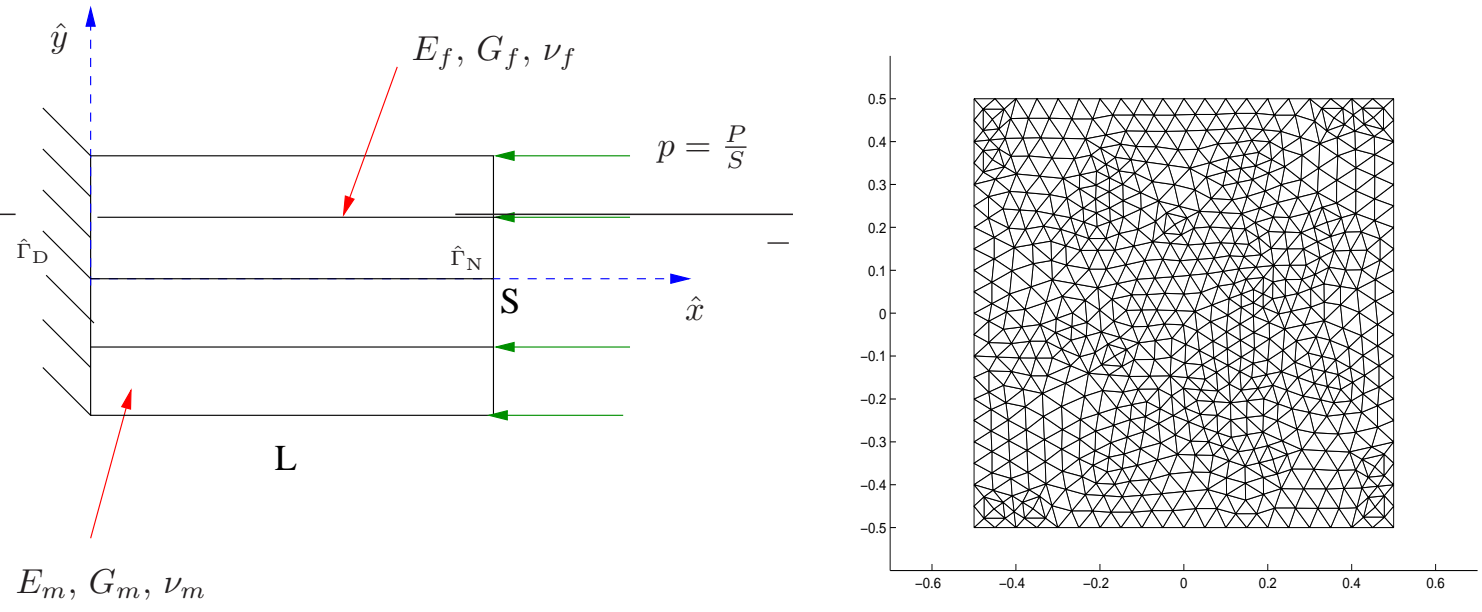

Figure 7: Schematic problem representation (left); mesh with 1296 elements (right).

- Geometry: the domain $\hat{\Omega}=(L, S)$. The parameter is the ratio $\frac{L}{S}$. 
- Physics: materials and their concentrations, so that coefficients $Q_{11}, Q_{12}, Q_{22}$ and $Q_{66}$ are varying with $E_{x}, E_{y}, \nu_{x y}, \nu_{y x}$ and $G_{x y}$, by (89) - (92), and are related to local coefficient of $E, G, \nu$ and $c$ of fibers and matrices by (79) - (82). Considering relative variations of fibers properties with respect to matrices the independent parameters are $\frac{E_{f}}{E_{m}}, \frac{c_{f}}{c_{m}}, \frac{\nu_{f}}{\nu_{m}}$.

The vector of parameters is: $\mu=\left\{\frac{L}{S}, \frac{E_{f}}{E_{m}}, \frac{c_{f}}{c_{m}}, \frac{\nu_{f}}{\nu_{m}}\right\} \in \mathcal{D}^{\mu} \subset \mathbb{R}^{P=4}$. On the boundary $\hat{\Gamma}$ of the domain $\hat{\Omega}$ concerning displacement we have

$$
\hat{u}=0 \quad \text { on } \hat{\Gamma}_{\mathrm{D}},
$$

and concerning stresses:

$$
\hat{\sigma}_{i j} \hat{\mathrm{e}}_{j}^{n}= \begin{cases}-\frac{P}{S} \hat{\mathrm{e}}_{i}^{n} & \text { on } \hat{\Gamma}_{\mathrm{N}} \\ 0 & \text { on } \hat{\Gamma} \backslash\left(\hat{\Gamma}_{\mathrm{D}} \cup \hat{\Gamma}_{\mathrm{N}}\right) .\end{cases}
$$

The mathematical formulation is: given $\mu \in \mathcal{D}^{\mu} \subset \mathbb{R}^{P=4}$, find $s(\mu)=\langle\hat{L}, \hat{u}\rangle$, where $\hat{u} \in \hat{Y}=\left(H^{1}(\hat{\Omega})\right)^{2}$ is the solution of $\langle\hat{\mathcal{A}} \hat{u}, \hat{v}\rangle=\langle\hat{F}, \hat{v}\rangle, \forall \hat{v} \in \hat{Y}$, knowing that $\langle\hat{L}, \hat{v}\rangle=$ $\langle\hat{F}, \hat{v}\rangle, \forall \hat{v} \in \hat{Y}$ (compliant),

$$
\begin{aligned}
\langle\hat{\mathcal{A}} \hat{w}, \hat{v}\rangle & =\int_{\hat{\Omega}} \frac{\partial \hat{v}_{i}}{\partial \hat{x}_{j}} \hat{C}_{i j k l} \frac{\partial \hat{w}_{k}}{\partial \hat{x}_{l}} \bar{\Omega} \quad \forall \hat{w}, \hat{v} \in \hat{Y}, \\
\langle\hat{F}, \hat{v}\rangle & =\int_{\hat{\Gamma}_{\mathrm{N}}} \hat{v}_{i} \hat{f}_{n} \hat{\mathrm{e}}_{i}^{n} \bar{\Gamma} \quad \forall \hat{v} \in \hat{Y},
\end{aligned}
$$

The geometrical transformation $\mathcal{G}(\hat{x})(\mu): \hat{\Omega} \longrightarrow \Omega$ is given by the following tensor

$$
\underline{G}(\mu)=\left[\begin{array}{cc}
\frac{1}{L} & 0 \\
0 & \frac{1}{S}
\end{array}\right]
$$

It follows

$$
\begin{aligned}
d \hat{\Omega} & =\operatorname{det} \underline{G}^{-1}(\mu) d \Omega=L S d \Omega \\
d \hat{\Gamma} & =\left|\underline{G}^{-1}(\mu) \underline{\mathrm{e}}^{t}\right| d \Gamma=S d \Gamma
\end{aligned}
$$

We rewrite the problem as

$$
\begin{aligned}
\langle\mathcal{A}(\mu) w, v\rangle=\langle\hat{\mathcal{A}} \hat{w}, \hat{v}\rangle & =\int_{\Omega} \frac{\partial w_{i}}{\partial x_{j}} C_{i j k l}(\mu) \frac{\partial v_{k}}{\partial x_{l}} d \Omega \quad \forall w, v \in Y, \\
\langle F(\mu), v\rangle=\langle\hat{F}, \hat{v}\rangle & =\int_{\Gamma_{\mathrm{N}}} f_{i}^{n}(\mu) v_{i} d \Gamma \quad \forall v \in Y .
\end{aligned}
$$


Developing the first term (100), by affine decomposition, and using the compact notation $\left(\frac{\partial w_{i}}{\partial x_{j}}=w_{i, j}\right)$, we get

$$
\begin{aligned}
\langle\mathcal{A}(\mu) w, v\rangle & =\frac{S}{L} Q_{11} \int_{\Omega} v_{1,1} w_{1,1} d \Omega+Q_{12} \int_{\Omega}\left(v_{2,2} w_{1,1}+v_{1,1} w_{2,2}\right) d \Omega+ \\
& +\frac{L}{S} Q_{66} \int_{\Omega} v_{1,2} w_{1,2} d \Omega+Q_{66} \int_{\Omega}\left(v_{1,2} w_{2,1}+v_{2,1} w_{1,2}\right) d \Omega+ \\
& +\frac{S}{L} Q_{66} \int_{\Omega} v_{2,1} w_{2,1} d \Omega+\frac{L}{S} Q_{22} \int_{\Omega} v_{2,2} w_{2,2} d \Omega .
\end{aligned}
$$

and by the affine decomposition we have:

$$
\begin{aligned}
\Theta^{1}(\mu) & =\frac{S}{L} Q_{11}, & \left\langle\mathcal{A}^{1} w, v\right\rangle & =\int_{\Omega} v_{1,1} w_{1,1} d \Omega, \\
\Theta^{2}(\mu) & =Q_{12}, & \left\langle\mathcal{A}^{2} w, v\right\rangle & =\int_{\Omega}\left(v_{2,2} w_{1,1}+v_{1,1} w_{2,2}\right) d \Omega, \\
\Theta^{3}(\mu) & =\frac{L}{S} Q_{66}, & \left\langle\mathcal{A}^{3} w, v\right\rangle & =\int_{\Omega} v_{1,2} w_{1,2} d \Omega, \\
\Theta^{4}(\mu) & =Q_{66}, & \left\langle\mathcal{A}^{4} w, v\right. & =\int_{\Omega}\left(v_{1,2} w_{2,1}+v_{2,1} w_{1,2}\right) d \Omega, \\
\Theta^{5}(\mu) & =\frac{S}{L} Q_{66}, & \left\langle\mathcal{A}^{5} w, v\right\rangle & =\int_{\Omega} v_{2,1} w_{2,1} d \Omega, \\
\Theta^{6}(\mu) & =\frac{L}{S} Q_{22}, & \left\langle\mathcal{A}^{6} w, v\right\rangle & =\int_{\Omega} v_{2,2} w_{2,2} d \Omega .
\end{aligned}
$$

\subsubsection{Numerical results}

We report in Figure 8 the numerical results representing horizontal and vertical displacement ( $u$ and $v$ respectively). In this case material is homogeneous $\left(E_{m}=E_{f}=E_{0}=10, \nu_{m}=\right.$ $\left.\nu_{f}=\nu_{0}=0.3, L=D=1, c_{m}=c_{f}=0.5\right)$. $E_{0}$ and $\nu_{0}$ are reference values.

We report now some results concerning the rapid convergence of the method: in Figure 9 we show the max and the average relative error $\left|e_{N}(\mu)\right|_{Y}$ testing a large number of different configurations (500) depending on 4 parameters and varying $N$. The basis is optimized according to the procedure described in Section 3. The points at each $N$ represent the error for a single configuration.

We provide also in Table 1 the values of coefficients for the two phases of the material: as fiber we have selected glass and an Epoxy resin for the matrix. In Table 2 we report the value of the global coefficient for the lamina after homogenization.

The values of parameters in the configurations we have tested was varying around the average value we reported in the tables. 

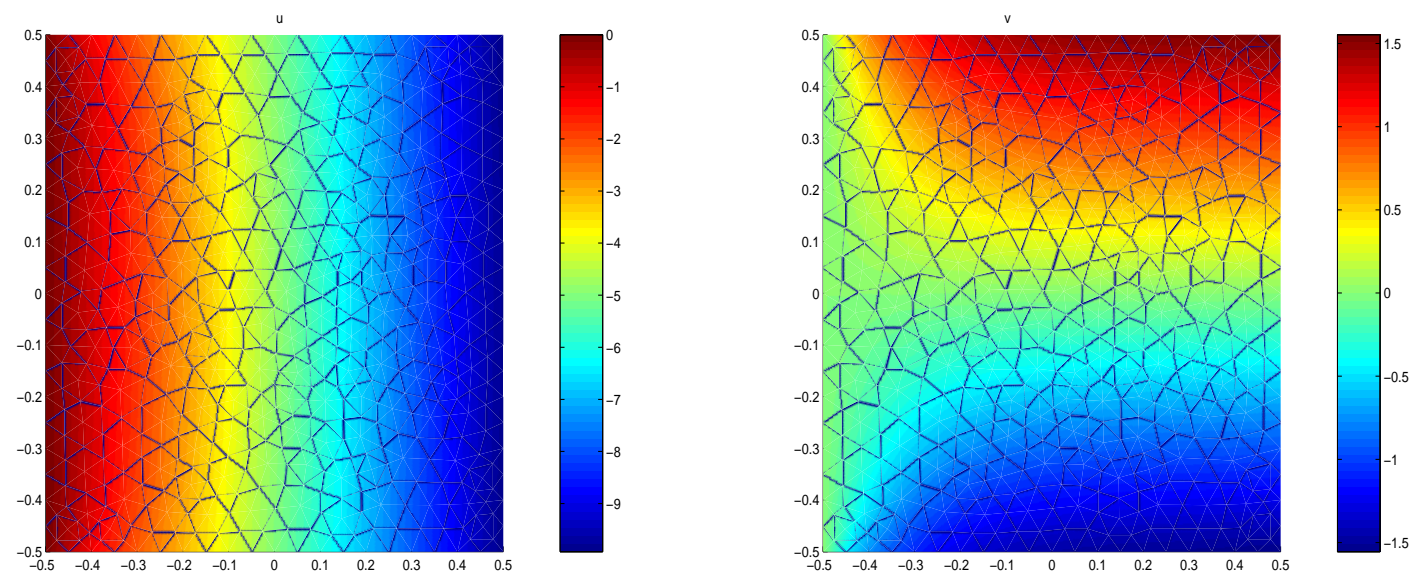

Figure 8: Displacement $u$ and $v$ for the orthotropic lamina $\left(\times 10^{-3} \mathrm{~m}\right)$.

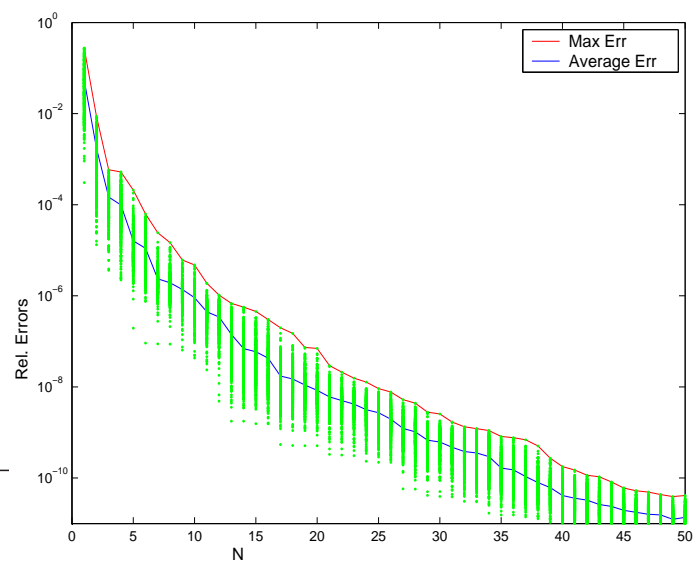

Figure 9: Max and average relative errors testing a large number of different configurations (500) depending on 4 parameters and varying $N$.

\subsection{Lamina in the general orthotropic case with varying geometry and angle of fibers}

We generalize the example of Section 6.1 by assuming that both the geometry and the fibers direction with respect to the load aligned with $x$ axis may vary. In Figure 10 we represent the schematic lamina under the approximation of plane stresses. In Figure 11 we report the parameters of our model, the mesh is the same as in Figure 7, while Figure 12 represents the direction of rotation.

The parameters are the following ones (Figure 11):

- Geometry: the domain $\hat{\Omega}=(L, S)$, with variable basis $L$ and height $S$.

- Physics: materials and their concentrations, so that coefficients $Q_{11}, Q_{12}, Q_{22}$ and $Q_{66}$ 


\begin{tabular}{|c|c|}
\hline Coefficients & Glass/Epoxy Resin \\
\hline$E_{f}$ & 70 \\
$\nu_{f}$ & 0.17 \\
$E_{m}$ & 2.85 \\
$\nu_{m}$ & 0.33 \\
$c_{f}$ & 0.66 \\
\hline
\end{tabular}

Table 1: Coefficients for glass fibers and epoxy resin. Young modulus is in [GPa].

\begin{tabular}{|c|c|}
\hline Coefficients & Reuss - Voigt \\
\hline$E_{x}$ & 47.16 \\
$E_{y}$ & 7.77 \\
$G_{x y}$ & 2.95 \\
$\nu_{x y}$ & 0.224 \\
\hline
\end{tabular}

Table 2: Coefficients computed using homogenization model by Reuss and Voigt. Young and tangential elastic modulus are in [GPa].

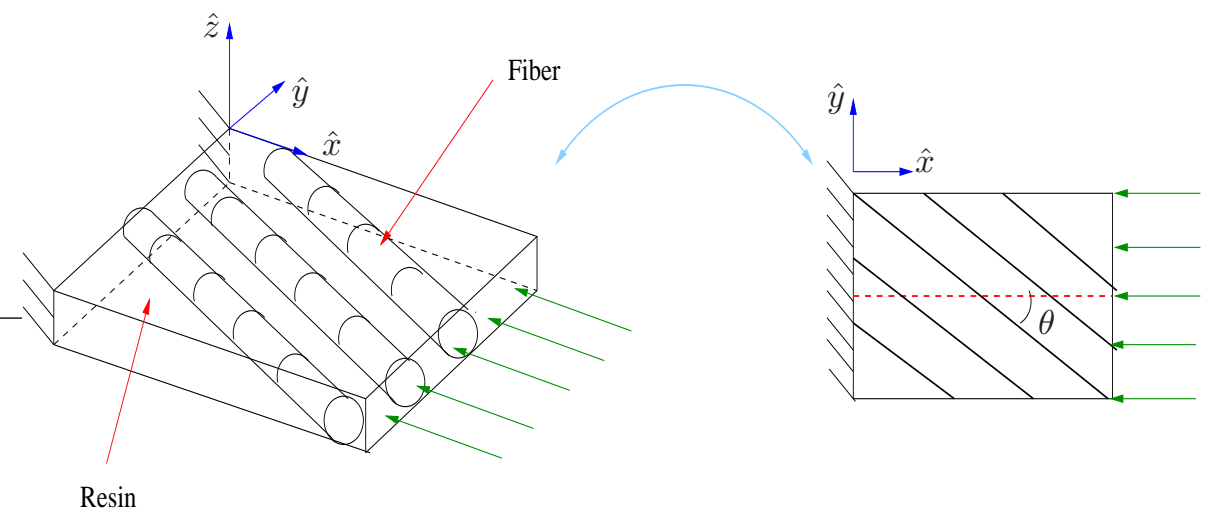

Figure 10: Orthotropic lamina: schematic representation.

vary, being related to $E_{x}, E_{y}, \nu_{x y}, \nu_{y x}$ and $G_{x y}$, by (89) - (92). Consequently, the local coefficient of $E, G, \nu$ and $c$ of fibers and matrices are given by (79)- (82). Considering relative variations of fibers properties with respect to matrices, the independent parameters are $\frac{E_{f}}{E_{m}}, \frac{c_{f}}{c_{m}}, \frac{\nu_{f}}{\nu_{m}}$. In addition we also consider the angle $\theta$ of fibers with respect to the main load direction.

The vector of parameters is: $\mu=\left\{\frac{L}{S}, \frac{E_{f}}{E_{m}}, \frac{c_{f}}{c_{m}}, \frac{\nu_{f}}{\nu_{m}}, \theta\right\} \in \mathcal{D}^{\mu} \subset \mathbb{R}^{P=5}$. The fibers direction is influencing the constitutive relationship between $\sigma$ and $\varepsilon$, see (86). We describe rotation of 


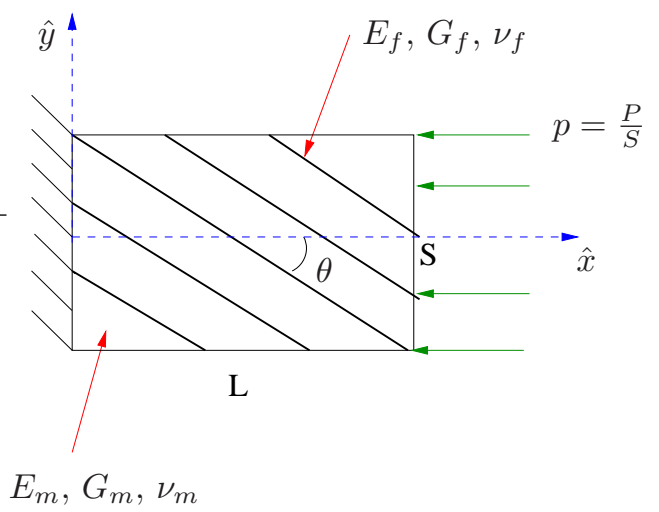

Figure 11: Orthotropic lamina: parameters.

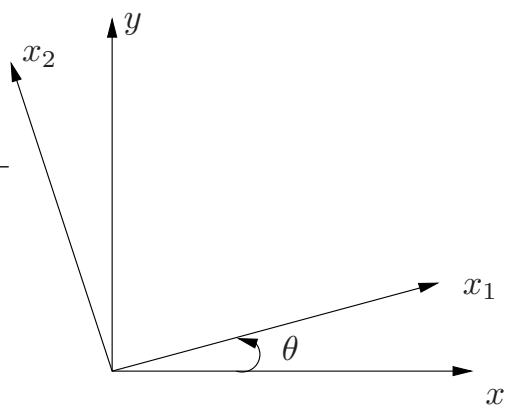

Figure 12: Introducing rotation in the system.

fibers as the rigid one shown in Figure 12 and given by

$$
\left\{\begin{array}{l}
x \\
y
\end{array}\right\}=\left[\begin{array}{cc}
\cos \theta & -\sin \theta \\
\sin \theta & \cos \theta
\end{array}\right]\left\{\begin{array}{l}
x_{1} \\
x_{2}
\end{array}\right\} .
$$

As described in [4], we may write:

$$
\left\{\begin{array}{c}
\hat{\sigma}_{11} \\
\hat{\sigma}_{22} \\
\hat{\sigma}_{12}
\end{array}\right\}=[T(\theta)]\left\{\begin{array}{c}
\hat{\sigma}_{x} \\
\hat{\sigma}_{y} \\
\hat{\tau}_{x y}
\end{array}\right\}, \quad\left\{\begin{array}{c}
\hat{\varepsilon}_{11} \\
\hat{\varepsilon}_{22} \\
\hat{\varepsilon}_{12} / 2
\end{array}\right\}=[T(\theta)]\left\{\begin{array}{c}
\hat{\varepsilon}_{x} \\
\hat{\varepsilon}_{y} \\
\hat{\varepsilon}_{x y}
\end{array}\right\},
$$

where the rotation tensor $[T(\theta)]$ is given by:

$$
[T(\theta)]=\left[\begin{array}{ccc}
\cos ^{2} \theta & \sin ^{2} \theta & 2 \sin \theta \cos \theta \\
\sin ^{2} \theta & \cos ^{2} \theta & -2 \sin \theta \cos \theta \\
-\sin \theta \cos \theta & \sin \theta \cos \theta & \cos ^{2} \theta-\sin ^{2} \theta
\end{array}\right] .
$$

Defining $[T(\theta)]^{-1}$ as the inverse of $[T(\theta)]$ and thanks to the properties of transformation 
matrix $[T(\theta)]$, we may verify:

$$
[T(\theta)]^{-1}=[T(-\theta)]=\left[\begin{array}{ccc}
\cos ^{2} \theta & \sin ^{2} \theta & -2 \sin \theta \cos \theta \\
\sin ^{2} \theta & \cos ^{2} \theta & 2 \sin \theta \cos \theta \\
\sin \theta \cos \theta & -\sin \theta \cos \theta & \cos ^{2} \theta-\sin ^{2} \theta
\end{array}\right]
$$

Therefore

$$
\left\{\begin{array}{c}
\hat{\sigma}_{x} \\
\hat{\sigma}_{y} \\
\hat{\tau}_{x y}
\end{array}\right\}=[T(-\theta)]\left\{\begin{array}{c}
\hat{\sigma}_{11} \\
\hat{\sigma}_{22} \\
\hat{\sigma}_{12}
\end{array}\right\}, \quad\left\{\begin{array}{c}
\hat{\varepsilon}_{x} \\
\hat{\varepsilon}_{y} \\
\hat{\varepsilon}_{x y}
\end{array}\right\}=[T(-\theta)]\left\{\begin{array}{c}
\hat{\varepsilon}_{11} \\
\hat{\varepsilon}_{22} \\
\hat{\varepsilon}_{12} / 2
\end{array}\right\} .
$$

Introducing Reuter matrices

$$
[R]=\left[\begin{array}{ccc}
1 & 0 & 0 \\
0 & 1 & 0 \\
0 & 0 & 2
\end{array}\right], \quad[R]^{-1}=\left[\begin{array}{ccc}
1 & 0 & 0 \\
0 & 1 & 0 \\
0 & 0 & \frac{1}{2}
\end{array}\right]
$$

and recalling that $\varepsilon_{x y}=\gamma_{x y} / 2$, thanks to (87), we can write:

$$
\left\{\begin{array}{c}
\hat{\sigma}_{x} \\
\hat{\sigma}_{y} \\
\hat{\tau}_{x y}
\end{array}\right\}=[T(-\theta)][Q][R][T(\theta)][R]^{-1}\left\{\begin{array}{c}
\hat{\varepsilon}_{x} \\
\hat{\varepsilon}_{y} \\
\hat{\gamma}_{x y}
\end{array}\right\}
$$

Thanks to (106), (107) and (109), we may verify

$$
[R][T(\theta)][R]^{-1}=\left[\begin{array}{ccc}
\cos ^{2} \theta & \sin ^{2} \theta & \sin \theta \cos \theta \\
\sin ^{2} \theta & \cos ^{2} \theta & -\sin \theta \cos \theta \\
-2 \sin \theta \cos \theta & 2 \sin \theta \cos \theta & \cos ^{2} \theta-\sin ^{2} \theta
\end{array}\right]=[T(-\theta)]^{T} .
$$

The rotation transformation is

$$
\left\{\begin{array}{c}
\hat{\sigma}_{x} \\
\hat{\sigma}_{y} \\
\hat{\tau}_{x y}
\end{array}\right\}=[\bar{Q}(\theta)]\left\{\begin{array}{c}
\hat{\varepsilon}_{x} \\
\hat{\varepsilon}_{y} \\
\hat{\gamma}_{x y}
\end{array}\right\}=\left[\begin{array}{ccc}
\bar{Q}_{11} & \bar{Q}_{12} & \bar{Q}_{16} \\
\bar{Q}_{12} & \bar{Q}_{22} & \bar{Q}_{26} \\
\bar{Q}_{16} & \bar{Q}_{26} & \bar{Q}_{66}
\end{array}\right]\left\{\begin{array}{c}
\hat{\varepsilon}_{x} \\
\hat{\varepsilon}_{y} \\
\hat{\gamma}_{x y}
\end{array}\right\}
$$

where

$$
[\bar{Q}(\theta)]=[T(-\theta)][Q][T(-\theta)]^{T}
$$


The components of matrix $\bar{Q}$ will be used in the general orthotropic case and are:

$$
\begin{aligned}
& \bar{Q}_{11}(\theta)=Q_{11} \cos ^{4} \theta+2\left(Q_{12}+2 Q_{66}\right) \sin ^{2} \theta \cos ^{2} \theta+Q_{22} \sin ^{4} \theta, \\
& \bar{Q}_{12}(\theta)=\left(Q_{11}+Q_{22}-4 Q_{66}\right) \sin ^{2} \theta \cos ^{2} \theta+Q_{12}\left(\sin ^{4} \theta+\cos ^{4} \theta\right), \\
& \bar{Q}_{22}(\theta)=Q_{11} \sin ^{4} \theta+2\left(Q_{12}+2 Q_{66}\right) \sin ^{2} \theta \cos ^{2} \theta+Q_{22} \cos ^{4} \theta, \\
& \bar{Q}_{16}(\theta)=\left(Q_{11}-Q_{12}-2 Q_{66}\right) \sin \theta \cos ^{3} \theta+\left(Q_{12}-Q_{22}+2 Q_{66}\right) \sin ^{3} \theta \cos \theta, \\
& \bar{Q}_{26}(\theta)=\left(Q_{11}-Q_{12}-2 Q_{66}\right) \sin ^{3} \theta \cos \theta+\left(Q_{12}-Q_{22}+2 Q_{66}\right) \sin \theta \cos ^{3} \theta, \\
& \bar{Q}_{66}(\theta)=\left(Q_{11}+Q_{22}-2 Q_{12}-2 Q_{66}\right) \sin ^{2} \theta \cos ^{2} \theta+Q_{66}\left(\sin ^{4} \theta+\cos ^{4} \theta\right),
\end{aligned}
$$

where $Q_{11}, Q_{12}, Q_{22}$ e $Q_{66}$ are defined in (89) - (92).

There are additional non-zero components due to the coupling between tangential (normal) stresses and normal (tangential) deformations which are typical of an anisotropic material. The lamina is always orthotropic and it is described by 4 physical coefficients $\left(E_{x}, E_{y}, \nu_{x y}\right.$ and $\left.G_{x y}\right)$. This is the general orthotropic case [4]. The model is the same of the previous section, only the term (100) is changing due to the rotation of fibers. By an affine decomposition we write:

$$
\begin{aligned}
\langle\mathcal{A}(\mu) w, v\rangle & =\frac{S}{L} \bar{Q}_{11} \int_{\Omega} v_{1,1} w_{1,1} d \Omega+\bar{Q}_{16} \int_{\Omega}\left(v_{1,2} w_{1,1}+v_{1,1} w_{1,2}\right) d \Omega+ \\
& +\frac{S}{L} \bar{Q}_{16} \int_{\Omega}\left(v_{2,1} w_{1,1}+v_{1,1} w_{2,1}\right) d \Omega+ \\
& +\bar{Q}_{12} \int_{\Omega}\left(v_{2,2} w_{1,1}+v_{1,1} w_{2,2}\right) d \Omega+ \\
& +\frac{L}{S} \bar{Q}_{66} \int_{\Omega} v_{1,2} w_{1,2} d \Omega+\bar{Q}_{66} \int_{\Omega}\left(v_{1,2} w_{2,1}+v_{2,1} w_{1,2}\right) d \Omega+ \\
& +\frac{L}{S} \bar{Q}_{26} \int_{\Omega}\left(v_{1,2} w_{2,2}+v_{2,2} w_{1,2}\right) d \Omega+ \\
& +\frac{S}{L} \bar{Q}_{66} \int_{\Omega} v_{2,1} w_{2,1} d \Omega+\bar{Q}_{26} \int_{\Omega}\left(v_{2,1} w_{2,2}+v_{2,2} w_{2,1}\right) d \Omega+ \\
& +\frac{L}{S} \bar{Q}_{22} \int_{\Omega} v_{2,2} w_{2,2} d \Omega
\end{aligned}
$$

By separation of parameter-dependent and parameter-independent contributions we have:

$$
\begin{aligned}
\Theta^{1}(\mu) & =\frac{S}{L} \bar{Q}_{11}, & \left\langle\mathcal{A}^{1} w, v\right\rangle & =\int_{\Omega} v_{1,1} w_{1,1} d \Omega, \\
\Theta^{2}(\mu) & =\bar{Q}_{16}, & \left\langle\mathcal{A}^{2} w, v\right\rangle & =\int_{\Omega}\left(v_{1,2} w_{1,1}+v_{1,1} w_{1,2}\right) d \Omega,
\end{aligned}
$$




$$
\begin{array}{rlrl}
\Theta^{3}(\mu) & =\frac{S}{L} \bar{Q}_{16}, & \left\langle\mathcal{A}^{3} w, v\right\rangle & =\int_{\Omega}\left(v_{2,1} w_{1,1}+v_{1,1} w_{2,1}\right) d \Omega, \\
\Theta^{4}(\mu) & =\bar{Q}_{12}, & \left\langle\mathcal{A}^{4} w, v\right\rangle & =\int_{\Omega}\left(v_{2,1} w_{1,1}+v_{1,1} w_{2,2}\right) d \Omega, \\
\Theta^{5}(\mu) & =\frac{L}{S} \bar{Q}_{66}, & \left\langle\mathcal{A}^{5} w, v\right\rangle & =\int_{\Omega} v_{1,2} w_{1,2} d \Omega, \\
\Theta^{6}(\mu) & =\bar{Q}_{66}, & \left\langle\mathcal{A}^{6} w, v\right\rangle & =\int_{\Omega}\left(v_{1,2} w_{2,1}+v_{2,1} w_{1,2}\right) d \Omega, \\
\Theta^{7}(\mu) & =\frac{L}{S} \bar{Q}_{26}, & \left\langle\mathcal{A}^{7} w, v\right\rangle & =\int_{\Omega}\left(v_{1,2} w_{2,2}+v_{2,2} w_{1,2}\right) d \Omega, \\
\Theta^{8}(\mu) & =\frac{S}{L} \bar{Q}_{66}, & \left\langle\mathcal{A}^{9} w, v\right\rangle & =\int_{\Omega}\left(v_{2,1} w_{2,2}+v_{2,2} w_{2,1}\right) d \Omega, \\
\Theta^{9}(\mu) & =\bar{Q}_{26}, & \left\langle\mathcal{A}^{10} w, v\right\rangle & =\int_{\Omega} v_{2,2} w_{2,2} d \Omega . \\
\Theta^{10}(\mu) & =\frac{L}{S} \bar{Q}_{22}, &
\end{array}
$$

The terms $\left\langle\mathcal{A}^{q} w, v\right\rangle$, with $q=1, \ldots, 10$, are computed once; the only terms varying are the ones depending on $\mu$, thus just $\Theta^{q}$ 's.

\subsubsection{Numerical results}

We report in Figures 13-16 some physical examples at different angles for fibers in the resin. Numerical results represent horizontal and vertical displacement ( $u$ and $v$ respectively). In this case the material has the following properties: $E_{m}=2.85 \cdot E_{0}, E_{f}=7.0 \cdot E_{0}, \nu_{m}=$ $3.3 \cdot \nu_{0}, \nu_{f}=1.7 \cdot \nu_{0}, L=D=1, c_{m}=0.67, c_{f}=0.33$, while $\theta=\frac{\pi}{6}, \frac{\pi}{4}, \frac{\pi}{3},-\frac{\pi}{4} . E_{0}=10, \nu_{0}=$ $0.10, \theta_{0}=0, L=S=1.0$ are reference values.

We report now some results concerning the rapid convergence of the method: in Figure 17 we show the max and the average relative error by testing a large number (500) of different configurations depending on 5 parameters and letting $N$ vary. The basis is optimized according to the procedure described in Section 3. The points (spots) at each $N$ represent the error for a single configuration. In Table 3 we report the reference value considered as average values for the parametrized configurations.

In Figure 18 we report the output (i.e. the average displacement of the loaded side) computed using RB when fibers angle and fibers concentration vary, and all other parameters are frozen.

As already seen the reduced basis method is very efficient for the solution of parametrized problems, we report Matlab cputime on the same architecture of Section 5.2 in Figure 19 (the comparisons are carried out by computing 100 and $150 \mathrm{FE}$ and RB solutions for the 

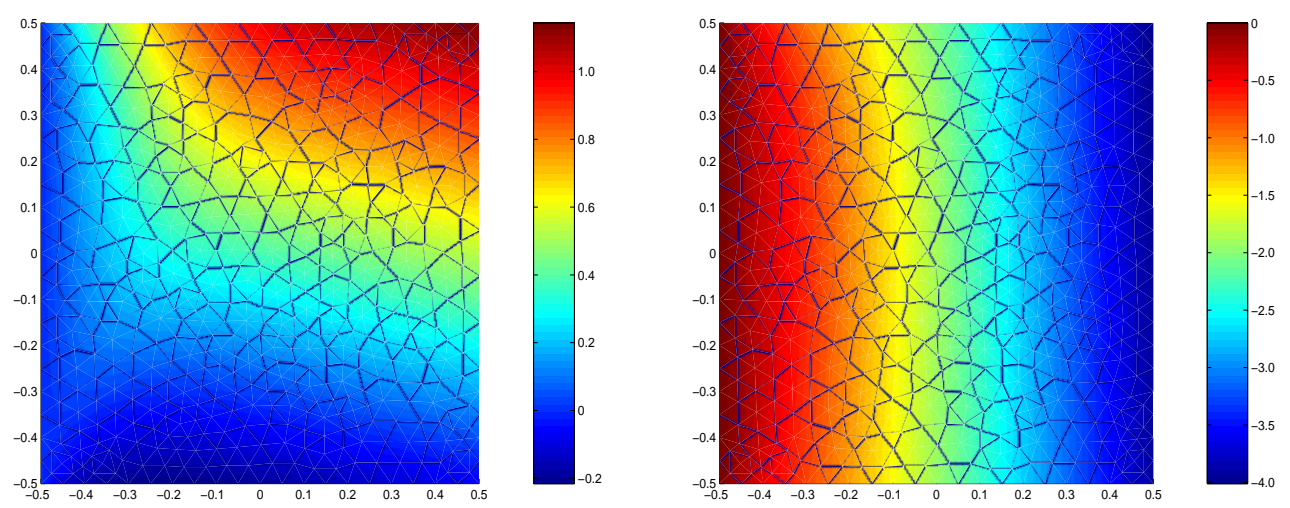

Figure 13: Displacement $v$ and $u$ for fibers with $\theta=\frac{\pi}{6},\left[\times 10^{-3} \mathrm{~m}\right]$.
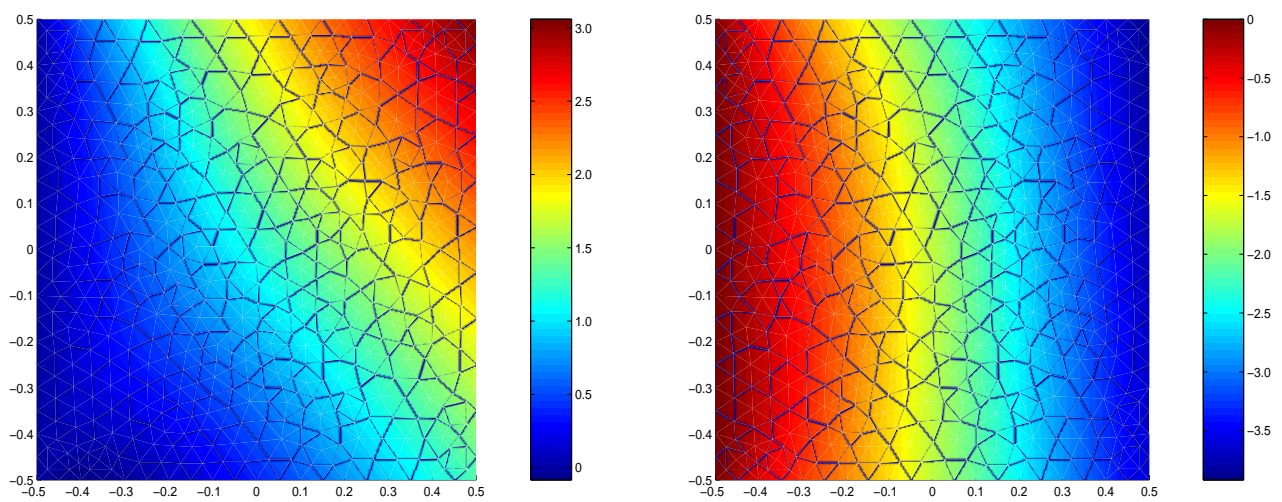

Figure 14: Displacement $v$ and $u$ for fibers with $\theta=\frac{\pi}{4},\left[\times 10^{-3} \mathrm{~m}\right]$.

same parameters combinations and for different dimensions $(N)$ of the RB space. RB are providing a computational saving of $97 \%$ with respect to $\mathrm{FE}$, without considering the time to build (and rebuild) meshes using FE or to assemble the basis for RB. The average time to get a FE solution is $0.78 s$ and $0.03 s$ for $\mathrm{RB}$ with $N=20$.

\section{Applications to isotropic damaged materials under harmonic loads}

Our last example is a problem with shear harmonic loads and isotropic materials in plane stress. We consider that the material has a damaged zone in the center, so that the stiffness of the central zone is reduced of a damage factor that we indicate with $\delta$ and the Young modulus is $\delta E$. Also in this case we deal with geometrical, physical and engineering parameters.

The plane stress formulation for an isotropic material is: 

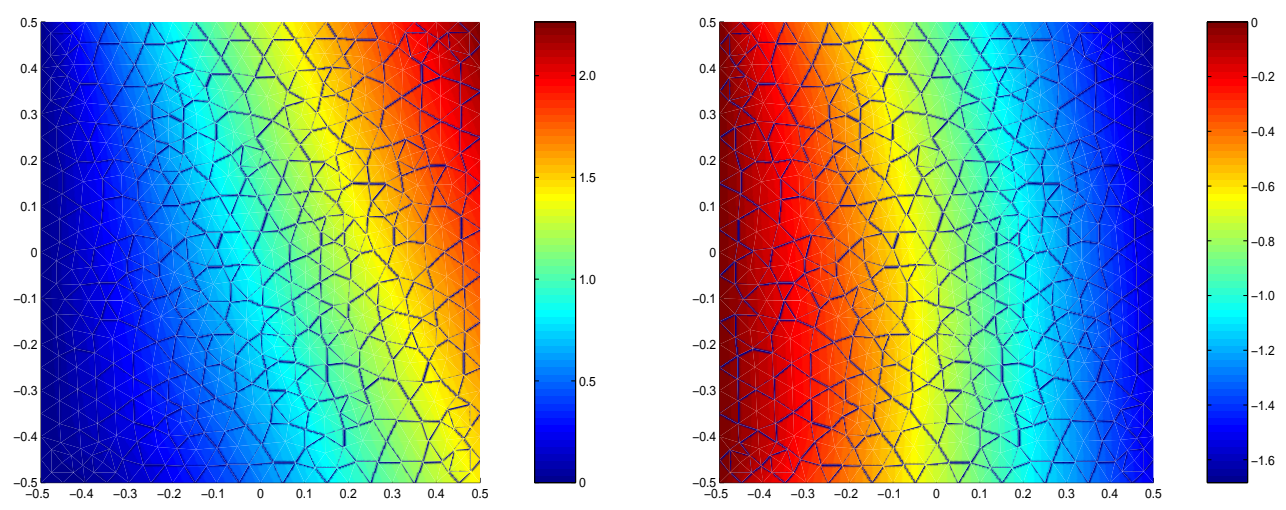

Figure 15: Displacement $v$ and $u$ for fibers with $\theta=\frac{\pi}{3},\left[\times 10^{-3} \mathrm{~m}\right]$.
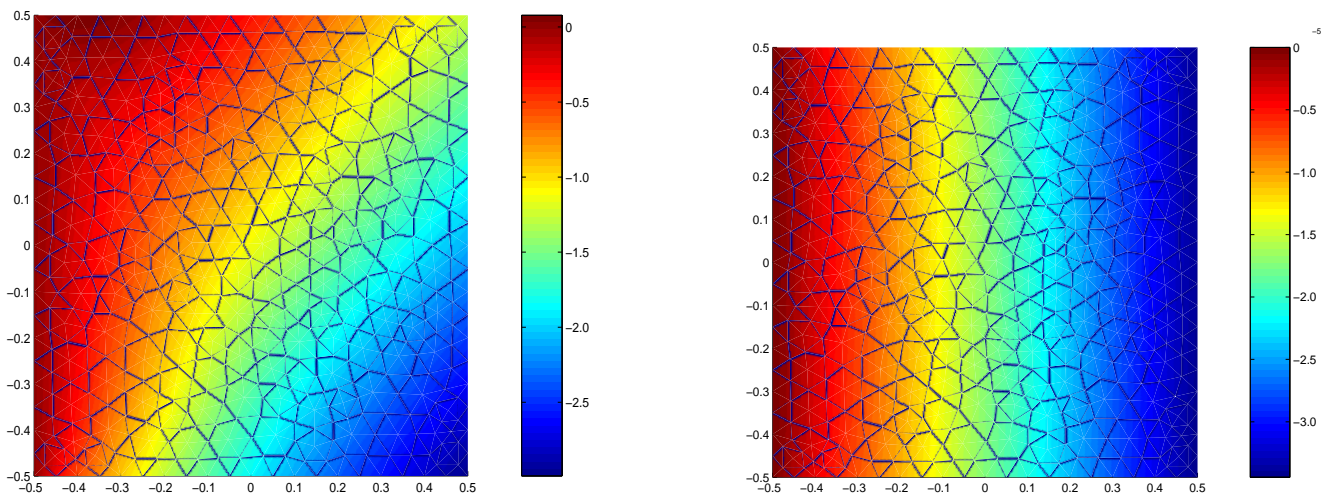

Figure 16: Displacement $u$ and $v$ for fibers with $\theta=-\frac{\pi}{4},\left[\times 10^{-3} \mathrm{~m}\right]$.

$$
\left\{\begin{array}{c}
\hat{\sigma}_{x} \\
\hat{\sigma}_{y} \\
\hat{\tau}_{x y}
\end{array}\right\}=\frac{E}{1-\nu^{2}}\left[\begin{array}{ccc}
1 & \nu & 0 \\
\nu & 1 & 0 \\
0 & 0 & \frac{(1-\nu)}{2}
\end{array}\right]\left\{\begin{array}{c}
\hat{\varepsilon}_{x} \\
\hat{\varepsilon}_{y} \\
\hat{\gamma}_{x y}
\end{array}\right\} .
$$

By the relationship $\sigma-\varepsilon$ of (116), we get the two Lamé constants $c_{1}$ and $c_{2}$ :

$$
c_{1}=\beta=\frac{E \nu}{(1+\nu)(1-\nu)}, c_{2}=G=\frac{E}{2(1+\nu)} .
$$

In Figure 20 we represent a lamina subject to shear harmonic loads on a side and clamped on the other one, and with a damaged inner zone.

As represented in Figure 21 the parameters are:

- Geometry: the domain $\hat{\Omega}$ is divided in 9 subdomains with variable dimensions: $\hat{\Omega}^{1}=$ $(S, V), \hat{\Omega}^{2}=(M, V), \hat{\Omega}^{3}=(L, V), \hat{\Omega}^{4}=(S, R), \hat{\Omega}^{5}=(M, R), \hat{\Omega}^{6}=(L, R), \hat{\Omega}^{7}=(S, Z)$, 


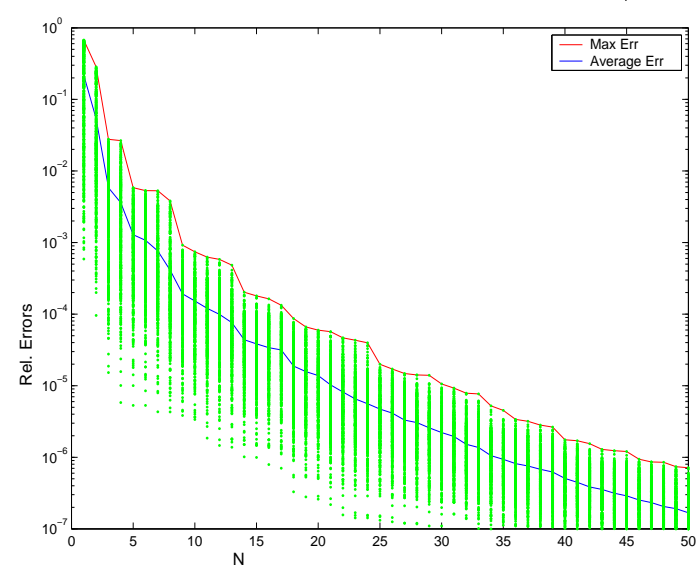

Figure 17: Max and average relative error by testing a large number of different configurations (500) depending on 5 parameters and varying $N$.

\begin{tabular}{|c|c|c|c|}
\hline Coefficients & Values & Coefficients & Values \\
\hline$E_{f}$ & 70.3 & $c_{f}$ & 0.84 \\
$\nu_{f}$ & 0.18 & $L$ & 1.23 \\
$E_{m}$ & 3.8 & $W$ & 1.22 \\
$\nu_{m}$ & 0.35 & $\theta$ & 13.6 \\
\hline
\end{tabular}

Table 3: Reference values considered, Young modulus was in [GPa], geometrical quantities in $[\mathrm{m}]$ and angles in degree.
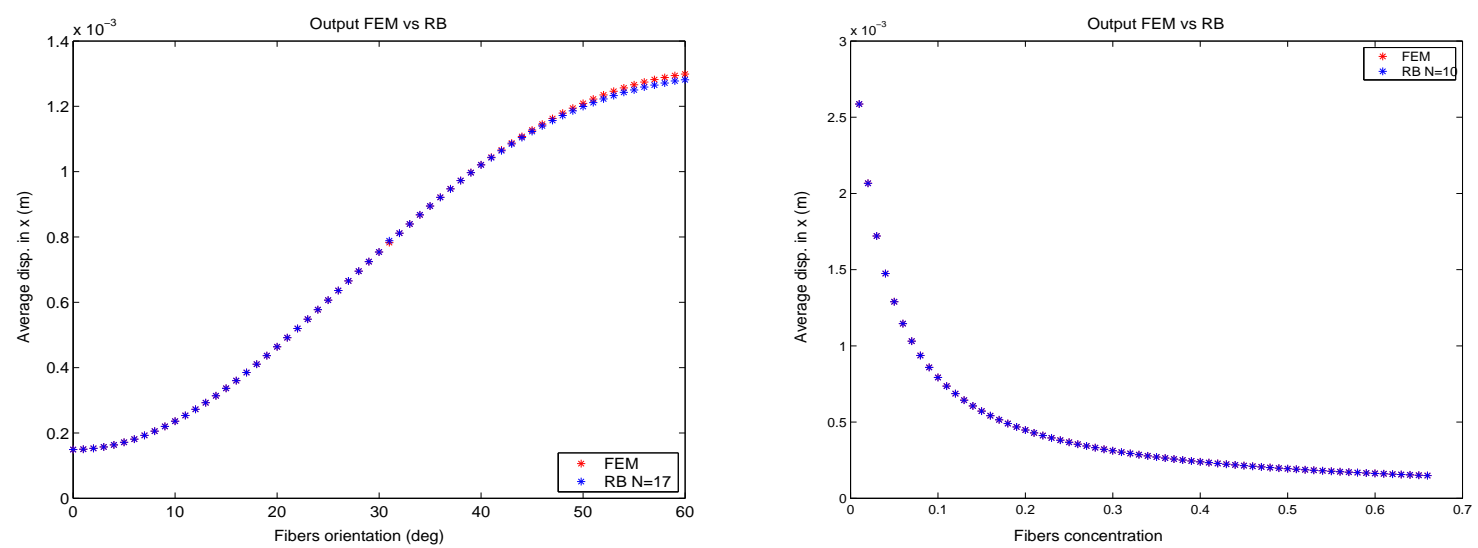

Figure 18: Output (average displacement of the loaded side) computed with FE and RB varying fibers orientation (left) and fibers concentration (volumetric fraction). 

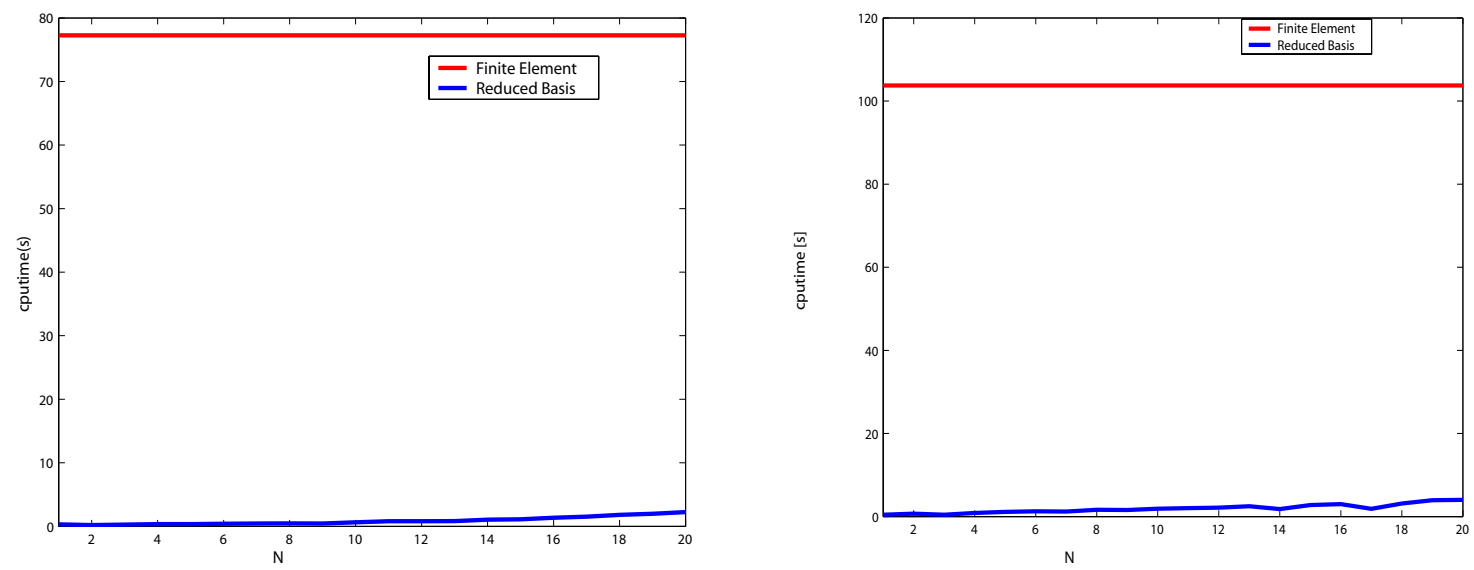

Figure 19: Comparison of computational times for 100 parametrized solutions (left) and 150 parametrized solutions (right) using finite element method and reduced basis mathod by increasing $N$.
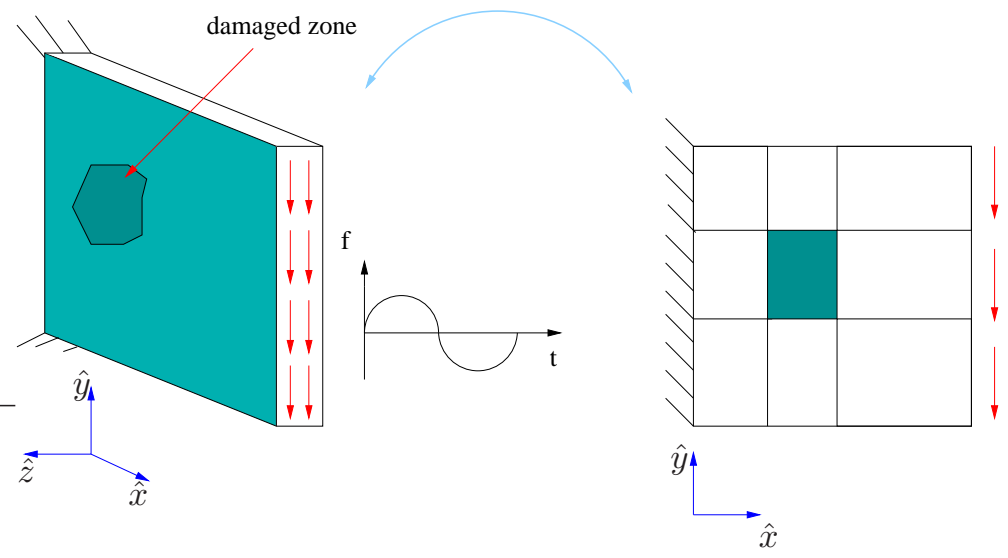

Figure 20: Isotropic lamina with a damaged inner zone under shear harmonic load and in plane stress approximation.

$\hat{\Omega}^{8}=(M, Z)$ and $\hat{\Omega}^{9}=(L, Z)$ such that:

$$
\left\{\begin{array}{l}
S+M+L=3 \\
Z+R+V=3
\end{array} .\right.
$$

Only 4 over 6 parameters are independent, for example $S, M, Z, R$. Both the dimension and the center of gravity of the damaged zone can vary.

- Physics: The material can change its properties $\beta$ and $G$, and we also introduce a damage factor $\delta \in[0.5,1]$ to create a variation in the Young modulus (stiffness).

- Engineering: loads $\hat{f}_{t}^{3}=-\frac{T}{V}, \hat{f}_{t}^{6}=-\frac{T}{R}$ and $\hat{f}_{t}^{9}=-\frac{T}{Z}$ are variable and they represent 

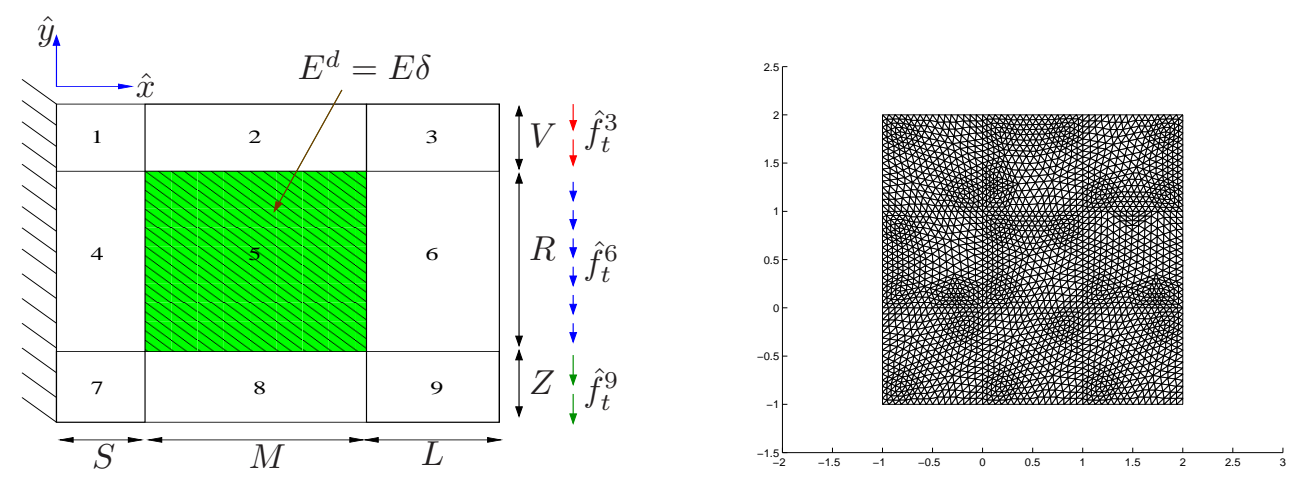

Figure 21: Schematic representation of the application (left); mesh used for FE calculation made up of 4896 elements (right).

the static load $T$, the shear force in each subdomain. The other parameter is $\omega$, the frequency of the load.

The vector of parameters is $\mu=\{S, M, Z, R, \beta, G, \delta, T, \omega\} \in \mathcal{D}^{\mu} \subset \mathbb{R}^{P=9}$.

Concerning boundary conditions, we have: $\hat{\Gamma}_{\mathrm{D}}=\hat{\Gamma}_{\mathrm{D}}^{1} \cup \hat{\Gamma}_{\mathrm{D}}^{4} \cup \hat{\Gamma}_{\mathrm{D}}^{7}$, so that:

$$
\hat{u}=0 \quad \text { on } \hat{\Gamma}_{\mathrm{D}},
$$

Concerning stresses, by denoting $\hat{\Gamma}_{\mathrm{N}}=\hat{\Gamma}_{\mathrm{N}}^{3} \cup \hat{\Gamma}_{\mathrm{N}}^{6} \cup \hat{\Gamma}_{\mathrm{N}}^{9}$, we get:

$$
\hat{\sigma}_{i j} \hat{\mathrm{e}}_{j}^{n}= \begin{cases}\hat{f}_{t}^{r} \hat{\mathrm{e}}_{i}^{t} & \text { on } \hat{\Gamma}_{\mathrm{N}}^{r} \\ 0 & \text { on } \hat{\Gamma} \backslash\left(\hat{\Gamma}_{\mathrm{D}} \cup \hat{\Gamma}_{\mathrm{N}}\right)\end{cases}
$$

where $r=3,6,9$. The mathematical formulation of the problem is: given $\mu \in \mathcal{D}^{\mu} \subset \mathbb{R}^{P=9}$, find $s(\mu)=\langle\hat{L}, \hat{u}\rangle$, where $\hat{u} \in \hat{Y}=\left(H^{1}(\hat{\Omega})\right)^{2}$ (see $(27)$ with $d=2$ ) is the solution of $\langle\hat{\mathcal{A}} \hat{u}, \hat{v}\rangle=\langle\hat{F}, \hat{v}\rangle, \forall \hat{v} \in \hat{Y}$, where $\langle\hat{L}, \hat{v}\rangle=\langle\hat{F}, \hat{v}\rangle, \forall \hat{v} \in \hat{Y}$ and

$$
\begin{aligned}
\langle\hat{\mathcal{A}} \hat{w}, \hat{v}\rangle & =\sum_{r=1}^{R} \int_{\hat{\Omega}^{r}}\left(\frac{\partial \hat{v}_{i}}{\partial \hat{x}_{j}} \hat{C}_{i j k l}^{r} \frac{\partial \hat{w}_{k}}{\partial \hat{x}_{l}}-\rho \omega^{2} \hat{w}_{i} \hat{v}_{i}\right) d \hat{\Omega} \quad \forall \hat{w}, \hat{v} \in \hat{Y}, \\
\langle\hat{F}, \hat{v}\rangle & =\sum_{r=1}^{R} \int_{\partial \hat{\Omega}^{r} \cap \hat{\Gamma}_{\mathrm{N}}} \hat{v}_{i} \hat{f}_{t}^{r} \hat{\mathrm{e}}_{i}^{t} d \hat{\Gamma} \quad \forall \hat{v} \in \hat{Y},
\end{aligned}
$$

with $R=9$.

The geometrical mapping from the original domain to the reference one is: $\mathcal{G}^{r}(\hat{x})(\mu)$ : $\hat{\Omega} \longrightarrow \Omega$ given by (38), where $\underline{g}=0$ and

$$
\underline{G}^{1}(\mu)=\left[\begin{array}{cc}
\frac{1}{S} & 0 \\
0 & \frac{1}{V}
\end{array}\right], \quad \underline{G}^{2}(\mu)=\left[\begin{array}{cc}
\frac{1}{M} & 0 \\
0 & \frac{1}{V}
\end{array}\right], \quad \underline{G}^{3}(\mu)=\left[\begin{array}{cc}
\frac{1}{L} & 0 \\
0 & \frac{1}{V}
\end{array}\right],
$$




$$
\begin{array}{lll}
\underline{G}^{4}(\mu)=\left[\begin{array}{cc}
\frac{1}{S} & 0 \\
0 & \frac{1}{R}
\end{array}\right], & \underline{G}^{5}(\mu)=\left[\begin{array}{cc}
\frac{1}{M} & 0 \\
0 & \frac{1}{R}
\end{array}\right], & \underline{G^{6}}(\mu)=\left[\begin{array}{cc}
\frac{1}{L} & 0 \\
0 & \frac{1}{R}
\end{array}\right], \\
\underline{G}^{7}(\mu)=\left[\begin{array}{cc}
\frac{1}{S} & 0 \\
0 & \frac{1}{Z}
\end{array}\right], & \underline{G^{8}}(\mu)=\left[\begin{array}{cc}
\frac{1}{M} & 0 \\
0 & \frac{1}{Z}
\end{array}\right], & \underline{G^{9}}(\mu)=\left[\begin{array}{cc}
\frac{1}{L} & 0 \\
0 & \frac{1}{Z}
\end{array}\right] .
\end{array}
$$

For the first subdomain (for example) we write:

$$
\begin{aligned}
d \hat{\Omega} & =\operatorname{det} \underline{G}^{1-1}(\mu) d \Omega=S V d \Omega, \\
d \hat{\Gamma} & =\left|\underline{G}^{1-1}(\mu) \underline{\mathrm{e}}^{t}\right| d \Gamma=V d \Gamma,
\end{aligned}
$$

while for the other subdomains we can replace $S$ and $V$ with the other corresponding geometrical parameters. By applying the domain transformation we write $\langle\mathcal{A}(\mu) w, v\rangle=\langle\hat{\mathcal{A}} \hat{w}, \hat{v}\rangle$ and $\langle F(\mu), v\rangle=\langle\hat{F}, \hat{v}\rangle$, where

$$
\begin{aligned}
\langle\mathcal{A}(\mu) w, v\rangle & =\sum_{r=1}^{R} \int_{\Omega^{r}}\left(\frac{\partial w_{i}}{\partial x_{j}} C_{i j k l}^{r}(\mu) \frac{\partial v_{k}}{\partial x_{l}}-\rho \omega^{2} w_{i} v_{i}\right) d \Omega \quad \forall w, v \in Y, \\
\langle F(\mu), v\rangle & =\sum_{r=1}^{R} \int_{\partial \Omega^{r} \cap \Gamma_{\mathrm{N}}} f_{i}^{t r}(\mu) v_{i} d \Gamma \quad \forall v \in Y,
\end{aligned}
$$

By applying an affine decomposition to each subdomain from (128) and the compact notation we get:

$$
\begin{aligned}
\langle\mathcal{A}(\mu) w, v\rangle & =\frac{V}{S}(2 G+\beta) \int_{\Omega^{1}} v_{1,1} w_{1,1} d \Omega+\beta \int_{\Omega^{1}}\left(v_{2,2} w_{1,1}+v_{1,1} w_{2,2}\right) d \Omega+ \\
& +\frac{S}{V} G \int_{\Omega^{1}} v_{1,2} w_{1,2} d \Omega+G \int_{\Omega^{1}}\left(v_{1,2} w_{2,1}+v_{2,1} w_{1,2}\right) d \Omega+ \\
& +\frac{V}{S} G \int_{\Omega^{1}} v_{2,1} w_{2,1} d \Omega+\frac{S}{V}(2 G+\beta) \int_{\Omega^{1}} v_{2,2} w_{2,2} d \Omega- \\
& -\rho \omega^{2} S V \int_{\Omega^{1}}\left(w_{1} v_{1}+w_{2} v_{2}\right) d \Omega+\ldots
\end{aligned}
$$

For the sake of notation we have just written the terms related with one subdomain: by replacing the quantities $(S, V)$ with $(V, L),(V, M),(R, S),(R, M),(R, L),(Z, S),(Z, M),(Z, L)$ we can get all the terms omitted. For example, just for one subdomain (number 5 ) where we have a damage factor $\delta$ we report parameter dependent quantities $\Theta^{q}(\mu)$ and parameter 
independent ones $\left\langle\mathcal{A}^{q} w, v\right\rangle$ :

$$
\begin{aligned}
\Theta^{1}(\mu) & =\frac{R}{M} \delta(2 G+\beta), & \left\langle\mathcal{A}^{1} w, v\right\rangle & =\int_{\Omega^{5}} v_{1,1} w_{1,1} d \Omega, \\
\Theta^{2}(\mu) & =\delta \beta, & \left\langle\mathcal{A}^{2} w, v\right\rangle & =\int_{\Omega^{5}}\left(v_{2,2} w_{1,1}+v_{1,1} w_{2,2}\right) d \Omega, \\
\Theta^{3}(\mu) & =\frac{M}{R} \delta G, & \left\langle\mathcal{A}^{3} w, v\right\rangle & =\int_{\Omega^{5}} v_{1,2} w_{1,2} d \Omega, \\
\Theta^{4}(\mu) & =\delta G, & \left\langle\mathcal{A}^{4} w, v\right\rangle & =\int_{\Omega^{5}}\left(v_{1,2} w_{2,1}+v_{2,1} w_{1,2}\right) d \Omega, \\
\Theta^{5}(\mu) & =\frac{R}{M} \delta G, & \left\langle\mathcal{A}^{5} w, v\right\rangle & =\int_{\Omega^{5}} v_{2,1} w_{2,1} d \Omega, \\
\Theta^{6}(\mu) & =\frac{M}{R} \delta(2 G+\beta), & \left\langle\mathcal{A}^{6} w, v\right\rangle & =\int_{\Omega^{5}} v_{2,2} w_{2,2} d \Omega, \\
\Theta^{7}(\mu) & =-\rho \omega^{2} M R, & \left\langle\mathcal{A}^{7} w, v\right\rangle & =\int_{\Omega^{5}}\left(w_{1} v_{1}+w_{2} v_{2}\right) d \Omega .
\end{aligned}
$$

The same procedure is applied to also to $\langle F(\mu), v\rangle$ :

$$
\begin{aligned}
\langle F(\mu), v\rangle & =\int_{\partial \Omega^{3} \cap \Gamma_{\mathrm{N}}} f_{2}^{t 3}(\mu) v_{2} d \Gamma+\int_{\partial \Omega^{6} \cap \Gamma_{\mathrm{N}}} f_{2}^{t 6}(\mu) v_{2} d \Gamma+\int_{\partial \Omega^{9} \cap \Gamma_{\mathrm{N}}} f_{2}^{t 9}(\mu) v_{2} d \Gamma= \\
& =-\int_{\partial \Omega^{3} \cap \Gamma_{\mathrm{N}}} \frac{T}{V} V v_{2} d \Gamma-\int_{\partial \Omega^{6} \cap \Gamma_{\mathrm{N}}} \frac{T}{R} R v_{2} d \Gamma-\int_{\partial \Omega^{9} \cap \Gamma_{\mathrm{N}}} \frac{T}{Z} Z v_{2} d \Gamma= \\
& =-T\left(\int_{\partial \Omega^{3} \cap \Gamma_{\mathrm{N}}} v_{2} d \Gamma+\int_{\partial \Omega^{6} \cap \Gamma_{\mathrm{N}}} v_{2} d \Gamma+\int_{\partial \Omega^{9} \cap \Gamma_{\mathrm{N}}} v_{2} d \Gamma\right)= \\
& =-T \int_{\Gamma_{\mathrm{N}}} v_{2} d \Gamma .
\end{aligned}
$$

used also as output of interest.

\subsection{Numerical results}

In Figure 22 we report the max and the average relative error using an optimized basis (left) and a random one (right) by testing a large number of parametrized configurations (represented by spots in the plot area) and increasing $N$. In Table 4 we report the range of variation for the parameters. We are interested here to maintain the coercivity of the operator, but the method can be extended also in the non-coercive case [18]. In Table 5 we report also some coefficients used to visualize an example in Figure 23 where we report shear stress and shear deformation $\gamma_{x y}$ : the damaged zone is visible in both stress and deformation plots. In Figure 24 we compare computational times for FE and RB solutions: also in this 


\begin{tabular}{|c|c|c|c|}
\hline Coefficients & Range & Coefficients & Range \\
\hline$E$ & $70 \div 80[G P a]$ & $S$ & $0.1 \div 1.1[\mathrm{~m}]$ \\
$\nu$ & $0.3 \div 0.4$ & $M$ & $0.1 \div 1.1[\mathrm{~m}]$ \\
$\delta$ & $0.5 \div 1.0$ & $Z$ & $0.1 \div 1.1[\mathrm{~m}]$ \\
$T$ & $(1.0 \div 2.0) \cdot 10^{6}[\mathrm{~N}]$ & $R$ & $0.1 \div 1.1[\mathrm{~m}]$ \\
$\omega$ & $90 \div 100^{\dagger}[\mathrm{Hz}]$ & & \\
\hline
\end{tabular}

Table 4: Ranges of parameters; $\nmid$ under the first resonance frequency.
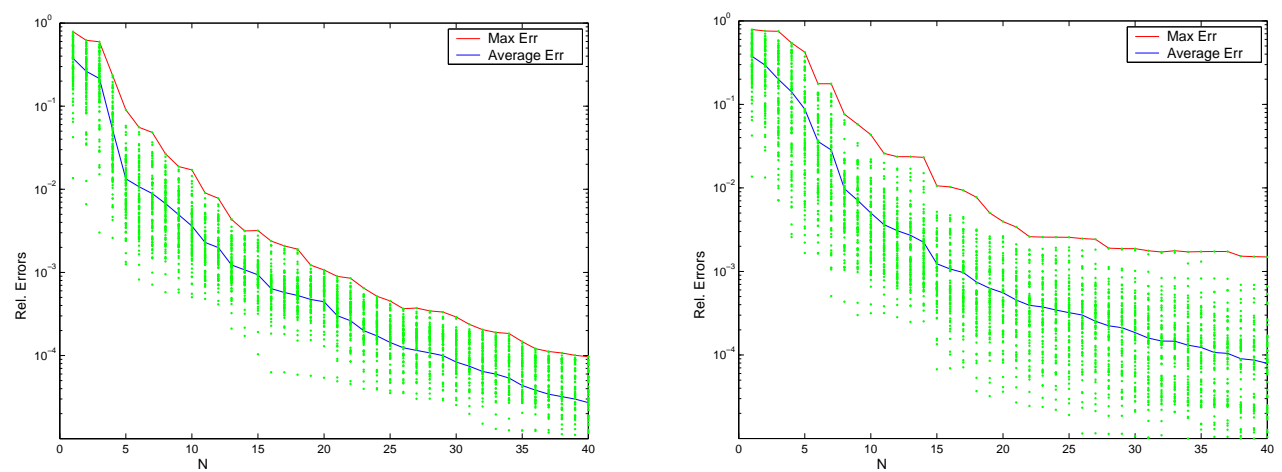

Figure 22: Max and average relative error seminorm is $H^{1}$ ) using an optimized basis (left) and a random one (right) by testing a large number of parametrized configuration (represented by spots in the plot area) and increasing $N$.
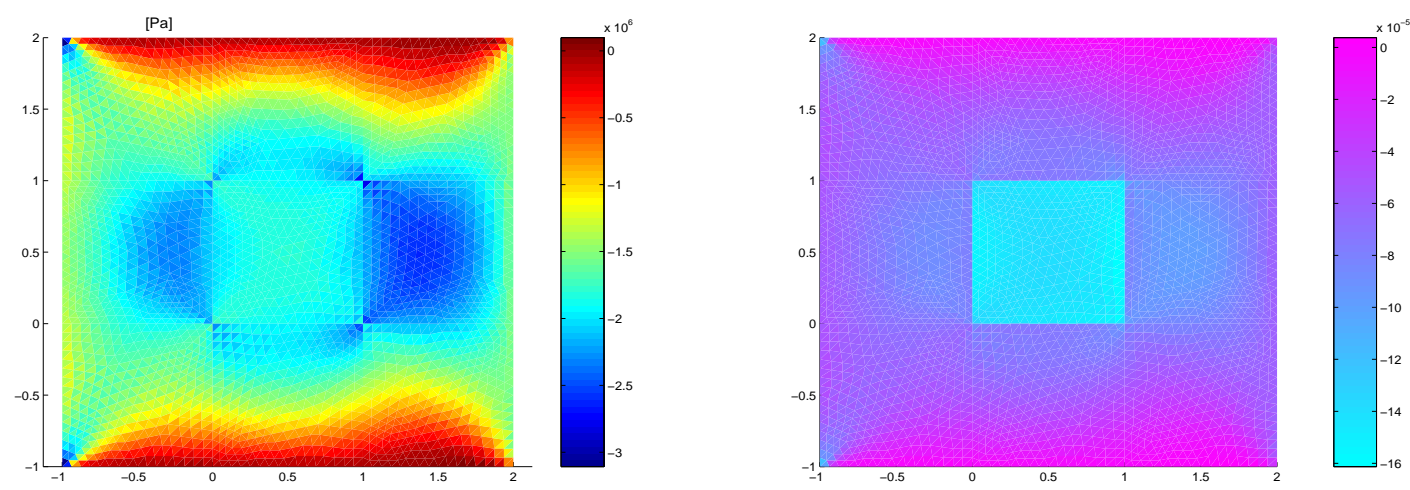

Figure 23: Shear stress $\tau_{x y}$ and shear deformation $\gamma_{x y}$ : the damaged zone is visible.

case we can guarantee computational time with a saving of $95 \%$ in the online calculation if compared with a FE solution. 


\begin{tabular}{|c|c|}
\hline Coefficients & Values \\
\hline$E$ & $70.48[G P a]$ \\
$\nu$ & 0.37 \\
$\delta$ & 0.5 \\
$T$ & $1.5 \cdot 10^{6}[\mathrm{~N}]$ \\
$\omega$ & $98[\mathrm{~Hz}]$ \\
\hline
\end{tabular}

Table 5: Values used as reference.
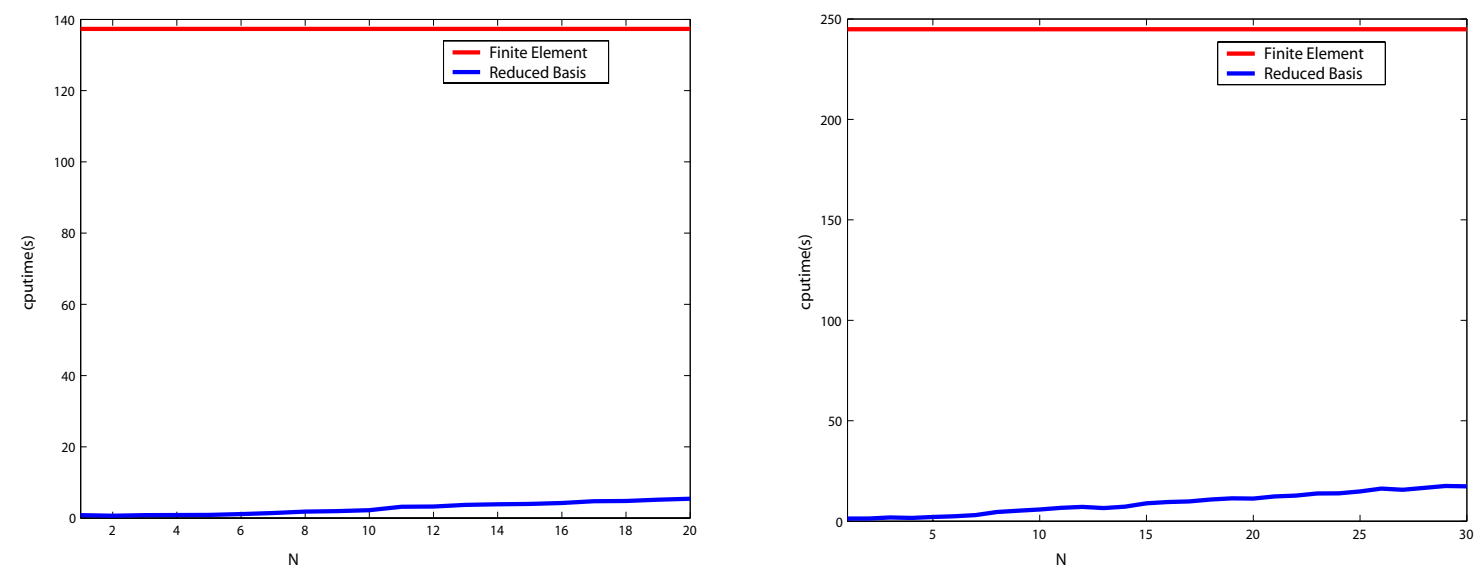

Figure 24: Comparison of computational times for 50 parametrized solutions (left) and 100 parametrized solutions (right) using finite element method and reduced basis mathod by increasing $N$.

\section{Conclusions}

We have provided some examples of applications of reduced basis methods in linear elasticity problems depending on many parameters of different kind (geometrical, physical, engineering) using linear elasticity approximation of plane strain and plane stress formulation. Both isotropic and orthotropic models have been considered, using homogenization for the latter to deal with composite materials. Reduced basis methods have confirmed a computational saving of about $95 \%$ with respect to a classical finite element formulation, not very suitable to solve parametrized problems in the real-time and many-query contexts. The possibility to treat with more complex outputs introducing a dual problem [24] and also with 3D applications [29] makes reduced basis methods quite promising. Another very important aspect is the certification of the errors in the reduced basis approximation by means of a posteriori error estimators, see for example [11]. 


\section{Acknowledgements}

We are sincerely grateful to Prof. A.T. Patera (MIT) for important suggestions, remarks and insights.

\section{References}

[1] K.A. Anagnostopoulos, A. Charalambopoulos, C.V. Massalas. On the investigation of elasticity equation for orthotropic materials and the solution of the associated scattering problem. Int. J. of Solids and Structures, 42 (2005), pp.6376-6408.

[2] P. G. Ciarlet. Mathematical Elasticity. Vol. I-II-III, North-Holland, Amsterdam (1988).

[3] R. M. Christensen. Mechanics of Composite Materials. Krieger, Malabar, Florida (1991).

[4] N. D. Cristescu, E. M. Craciun, E. Soos. Mechanics of Elastic Composites. Ed. Chapman \& Hall / CRC, Boca Raton, Florida (2004).

[5] R. Dautray, J. L. Lions. Mathematical Analysis and Numerical Methods for Science and Technology. Vol. I, Springer, Berlino (2000).

[6] P. L. Gould. Introduction to Linear Elasticity. Springer - Verlag, New York, Berlin, Heidelberg, Tokyo, (1983).

[7] G. Grimvall. Thermophysical Properties of Materials. North-Holland, Amsterdam (1999).

[8] Z. Hashin. Analysis of composites materials. Journal of Applied Mechanics (1983), Vol. 50, pp. $481-505$.

[9] Z. Hashin, S. Shtrikman. A variational approach to the theory of the elastic behaviour of multiphase materials. Journal Mech. Phys. Solids (1963), Vol. 11, pp. 127 - 140.

[10] D. B. P. Hyunh, A. T. Patera. Reduced-Basis Approximation and A Posteriori Error Estimation for Stress Intensity Factors. Int. J. Num. Meth. Eng. (2007), Vol. 72, No. 10, pp.1219 - 1259 .

[11] D. B. P. Huynh, G. Rozza, S. Sen A. T. Patera. A successive constraint linear optimization method for lower bounds of parametric coercivity and inf-sup stability constants. C. R. Acad. Sci. Paris, Analyse Numérique (2007), Vol. 345, No. 8, pp.473-478.

[12] D. B. P. Hyunh, Reduced-Basis Approximation and Application to Fracture and Inverse Analysis, PhD Thesis Singapore-MIT Alliance, National University of Singapore (2007).

[13] H. Leipholz. Theory of Elasticity. Noordhoff International Publishing, Leyden (1974).

[14] R. Leis, Initial Boundary Value Problems in Mathematical Physics, J. Wiley, Neyork, and B.G. Teubner, Stuttgart, 1996. 
[15] A. E. H. Love. A Treatise on the Mathematical Theory of Elasticity. Dover Publications, New York (1944).

[16] Y. Maday, A. T. Patera, G. Turinici. A priori convergence theory for reduced-basis approximation of single-parameter symmetric coercive elliptic partial differential equations. Journal of Scientific Computing (2002), Vol. 17, No. 1-4, pp. 437 - 446.

[17] D. A. Nagy. Modal representation of geometrically nonlinear behavior by the finite element method. Computers \& Structures (1978), Vol. 10, pp. 683 - 688.

[18] N. C. Nguyen. Reduced Basis Approximations and A Posteriori Error Bounds for Nonaffine and Nonlinear Partial Differential Equations: Application to Inverse Analysis. PhD Thesis, Singapore-MIT Alliance (2005).

[19] N. C. Nguyen, K. Veroy and A. T. Patera. Certified Real-Time Solution of Parametrized Partial Differential Equations. In Handbook of Materials Modeling, S. Yip Ed., Springer, NewYork, 2005, pp.1523-1558.

[20] A. K. Noor and J. M. Peters. Reduced basis technique for nonlinear analysis of structures. AIAA Journal (1980), Vol. 18, No. 4, Article No. 79 - 0747R, pp. 455 - 462.

[21] A. K. Noor and J. M. Peters. Tracing post-limit point paths with reduced basis technique. Computer Methods in Applied Mechanics and Engineering (1981), Vol. 28, pp. 217 240.

[22] A. K. Noor and J. M. Peters. Bifurcation and post-buckling analysis of laminated composite plates via reduced basis technique. Computer Methods in Applied Mechanics and Engineering (1981), Vol. 29, pp. 271 - 295.

[23] A. K. Noor. On making large nonlinear problems small. Computer Methods in Applied Mechanics and Engineering (1981), Vol. 34, pp. 955 - 985.

[24] A.T. Patera, G. Rozza. Reduced Basis Approximation and A Posteriori Error Estimation for Parametrized PDEs, MIT-Pappalardo Graduate Monographs in Mechanical Engineering Series, in progress, 2007. Available at http://augustine.mit.edu/methodology/methodology_book.htm.

[25] C. Prud'homme, D. V. Rovas, K. Veroy, L. Machiels, Y. Maday, A. T. Patera, G. Turinici. Reliable real-time solution of parametrized partial differential equations: reduced-basis output bound methods. J. Fluids Engineering (2002), Vol. 124, N.1, pp. 70 $-80$.

[26] A. Quarteroni, A. Valli. Numerical Approximation of Partial Differential Equations. Springer - Verlag, Berlin (1994).

[27] A. Quarteroni, R. Sacco, F. Saleri. Numerical Mathematics. Springer, New York (2006). 
[28] G. Rozza. Reduced basis methods for elliptic equations in sub-domains with a posteriori error bounds and adaptivity. Applied Numerical Mathematics (2005), Vol. 55, No. 4, pp. $403-424$.

[29] G. Rozza, D. B. P. Huynh, A.T. Patera. Reduced basis approximation and a posteriori error estimation for affinely parametrized elliptic coercive partial differential equations. Archive of Computational Methods in Engineering (2008), Vol. 15, No. 3.

[30] G. Rozza, K. Veroy. On the stability of reduced basis method for Stokes equations in parametrized domains. Computer Methods in Applied Mechanics and Engineering (2007), Vol.196, No. 7, pp.1244-1260.

[31] M.H. Sadd, Elasticity: Theory, Applications and Numerics, Elsevier, Boston, 2005.

[32] I. S. Sokolnikoff. Mathematical Theory of Elasticity. Krieger, Malabar, Florida (1983).

[33] K. Veroy. Reduced-Basis Methods Applied to Problems in Elasticity: Analysis and Applications. PhD Thesis, MIT (2003).

[34] K. Veroy, C. Prud'homme, D. Rovas, A.T. Patera. A posteriori error bounds for reducedbasis approximation of parametrized noncoercive and nonlinear elliptic partial differential equations. AIAA American Institute of Aeronautics and Astronatics. Paper 2003-3847 (2003).

[35] K. Washizu. Variational Methods in Elasticity and Plasticity. Ed. Oxford: Pergamon Press, Third edition (1982). 